\title{
Products of Reflections and Triangularization of Bilinear Forms
}

\author{
Jacques Helmstetter ${ }^{1}$
}

${ }^{1}$ Université de Grenoble-Alpes. France

Correspondence: Jacques Helmstetter, Université de Grenoble-Alpes, Saint-Martin d'Hères, France. E-mail: jacques.helmstetter@univ-grenoble-alpes.fr

Received: December 2, 2016 Accepted: January 24, 2017 Online Published: March 5, 2017

doi:10.5539/jmr.v9n2p18 URL: https://doi.org/10.5539/jmr.v9n2p18

\begin{abstract}
The present article is motivated by the theorem of Cartan-Dieudonné which states that every orthogonal transformation is a product of reflections. Its purpose is to determine, for each orthogonal transformation, the minimal number of factors in a decomposition into a product of reflections, and to propose an effective algorithm giving such a decomposition. With the orthogonal transformations $g$ of a quadratic space $(V, q)$, it associates couples $(S, \phi)$ where $S$ is a subspace of $V$, and $\phi$ an non-degenerate bilinear form on $S$ such that $\phi(y, y)=q(y)$ for every $y$ in $S$. In general, the minimal decompositions of $g$ into a product of reflections correspond to the bases of $S$ in which the matrix of $\phi$ is lower triangular. Therefore, we need an algorithm of triangularization of bilinear forms. Affine isometries are also taken into consideration.
\end{abstract}

Keywords: orthogonal transformations, bilinear forms.

Let $V$ be a vector space of finite dimension $n$ over a field $K, q$ a quadratic form on $V$ which is momentarily assumed to be non-degenerate, and $\mathrm{O}(V, q)$ the group of its orthogonal transformations. Since the characteristic of $K$ may be 2, the associated bilinear form $\mathrm{b}_{q}$ is defined in this way:

$$
\forall x, y \in V, \quad \mathrm{~b}_{q}(x, y)=q(x+y)-q(x)-q(y) ;
$$

thus $\mathrm{b}_{q}(x, x)=2 q(x)$ for all $x$. Every non-isotropic vector $v \in V$ determines a reflection $\mathrm{R}(v)$ :

$$
\forall x \in V, \quad \mathrm{R}(v)(x)=x-\frac{\mathrm{b}_{q}(x, v)}{q(v)} v .
$$

The theorem of Cartan-Dieudonné (see (Dieudonné, 1958)) states that every $g \in \mathrm{O}(V, q)$ is a product of reflections, where the number of reflections is $\leq n$. Nevertheless, there are exceptions when the field $K$ is isomorphic to $\mathbb{Z} / 2 \mathbb{Z}$. When $q$ is anisotropic (for instance when $K=\mathbb{R}$ and $q$ is euclidean), it is easy to prove that the minimal number of reflections for a particular $g$ is the dimension of $\operatorname{im}(g-\mathbf{1})$, the image of $g-\mathbf{1}_{V}$ (where $\mathbf{1}_{V}$ is the identity mapping of $V$, also denoted by $\mathbf{1}$ if this short notation is clear enough). The determination of this minimal number is much more difficult when there are non-zero isotropic vectors $x$ (such that $q(x)=0$ ). Here this minimal number proves to be the dimension of $\operatorname{im}(g-\mathbf{1})$ when it is not totally isotropic, and $\operatorname{dim}(\operatorname{im}(g-\mathbf{1}))+2$ when it is totally isotropic; because of the above mentioned exceptions, $K$ is assumed not to be isomorphic to $\mathbb{Z} / 2 \mathbb{Z}$.

I first tackled this problem with the Clifford algebra $\mathrm{Cl}(V, q)$ (the associative and unital algebra generated by the elements $x$ of $V$ with the relations $x^{2}=q(x)$ ); but in this article, contrary to (Helmstetter 2017), I present only the part of my research that can be explained without mentioning Clifford algebras. Nevertheless, the Clifford algebras suggested new points of view and new definitions that I shall explain at once. Firstly, the hypothesis that $q$ is non-degenerate has been removed, because it causes a dreadful loss of effectiveness in the treatment of Clifford algebras. We must pay attention to $\operatorname{ker}\left(\mathrm{b}_{q}\right)$, the subspace of all $x \in V$ such that $\mathrm{b}_{q}(x, y)=0$ for all $y \in V$, and to $\operatorname{ker}(q)$, the subspace of all $x \in \operatorname{ker}\left(\mathrm{b}_{q}\right)$ such that $q(x)=0$; since $\mathrm{b}_{q}(x, x)=2 q(x)$, the equality $\operatorname{ker}(q)=\operatorname{ker}\left(\mathrm{b}_{q}\right)$ holds whenever the characteristic of $K$ is $\neq 2$. When $\operatorname{ker}(q) \neq \operatorname{ker}\left(\mathrm{b}_{q}\right), q$ is said to be defective. Secondly, we must distinguish $\operatorname{Iso}(V, q)$, the group of isometries of $(V, q)$, and its subgroup $\mathrm{O}(V, q)$, the group of orthogonal transformations; a linear transformation $g$ of $V$ is an isometry if (by definition) $q(g(x))=q(x)$ for all $x \in V$; an isometry $g$ is an orthogonal transformation if $\operatorname{ker}(g-\mathbf{1}) \supset \operatorname{ker}\left(\mathbf{b}_{q}\right)$. For instance, every reflection $\mathrm{R}(v)$ is an orthogonal transformation, and $\operatorname{im}(\mathrm{R}(v)-\mathbf{1})$ is the line spanned by $v$ (except when $q$ is defective and $\left.v \in \operatorname{ker}\left(\mathrm{b}_{q}\right)\right)$. A linear transformation $g$ is an isometry if and only if it extends to an automorphism of $\mathrm{Cl}(V, q)$; it is an orthogonal transformation if and only if it extends to a twisted inner automorphism of $\mathrm{Cl}(V, q)$ according to this definition which involves the parity gradation of $\mathrm{Cl}(V, q)$ : the twisted inner automorphism determined by an invertible, even or odd element $a \in \mathrm{Cl}(V, q)$ is $b \longmapsto a b a^{-1}$ if $a$ or $b$ is even, $b \longmapsto-a b a^{-1}$ if $a$ and $b$ are odd. Thirdly, every orthogonal transformation $g$ can be determined by a couple $(S, \phi)$ where $S$ is a subspace of $V$ containing $\operatorname{im}(g-\mathbf{1})$, and $\phi$ is a non-degenerate bilinear form on $S$ such that $\phi(y, y)=q(y)$ for all $y \in S$. Since we shall meet plenty of such couples 
$(S, \phi)$, I propose to call them transformers of $(V, q)$. When $q$ is non-degenerate (in other words, $\left.\operatorname{ker}\left(\mathrm{b}_{q}\right)=0\right)$, then $g$ admits only one transformer $(S, \phi)$, and $S=\mathrm{im}(g-\mathbf{1})$. But in other cases, there may be plenty of transformers over each $g \in \mathrm{O}(V, q)$, sometimes of various dimensions; therefore, the determination of their minimal dimension is important:

$$
\text { minimal } \operatorname{dim}(S)=\operatorname{dim}(\operatorname{im}(g-\mathbf{1}))+\operatorname{dim}(\operatorname{im}(g-\mathbf{1}) \cap \operatorname{ker}(q)) .
$$

This minimal dimension $s$ gives the minimal number of factors in a decomposition of $g$ into a product of reflections; it is $s$ when $q$ admits a minimal-dimensional transformer $(S, \phi)$ that is not totally isotropic; in the other cases, it is $s+2$ (only $s+1$ if $q$ is defective).

The quadratic space $(V, q)$ is said to be embedded in $(W, \tilde{q})$ if there is an injective linear mapping $f: V \rightarrow W$ such that $\tilde{q}(f(x))=q(x)$ for all $x$; for convenience, $V$ will be treated as a subspace of $W$, and $\tilde{q}$ as an extension of $q$. Such an embedding is especially interesting if $\tilde{q}$ is non-degenerate; indeed, we shall realize that an isometry $g$ of $(V, q)$ is an orthogonal transformation if and only if it extends to an orthogonal transformation $\tilde{g}$ of $(W, \tilde{q}) \operatorname{such} \operatorname{that} \operatorname{im}\left(\tilde{g}-\mathbf{1}_{W}\right) \subset V$; in other words, $\mathrm{O}(V, q)$ is the image of the subgroup of all $\tilde{g} \in \mathrm{O}(W, \tilde{q})$ such that $\operatorname{im}\left(\tilde{g}-\mathbf{1}_{W}\right) \subset V$; the image of each $\tilde{g}$ is its restriction to $V$; moreover, the suitable extensions $\tilde{g}$ of $g$ are in bijection with the transformers $(S, \phi)$ over $g$.

Example. When $q$ is the null quadratic form on $V$, then $\operatorname{Iso}(V, q)$ is the linear group $\operatorname{GL}(V)$ whereas $\mathrm{O}(V, q)$ is the trivial group $\left\{\mathbf{1}_{V}\right\}$. There is a non-degenerate embedding $(W, \tilde{q})$ where $W$ is the direct sum of $V$ and the dual space $V^{*}$, and where $\tilde{q}(x, \ell)=\ell(x)$ for all $x \in V$ and all $\ell \in V^{*}$. Every $g \in \mathrm{GL}(V)$ has extensions $\tilde{g}$ in $\mathrm{O}(W, \tilde{q})$, and there is a canonical extension $(x, \ell) \longmapsto\left(g(x), \ell \circ g^{-1}\right)$; but $\operatorname{im}\left(\tilde{g}-\mathbf{1}_{W}\right)$ is not contained in $V$ if $g \neq \mathbf{1}_{V}$; indeed, Lemma 1.2 (here below) shows that the conditions $\operatorname{im}\left(\tilde{g}-\mathbf{1}_{W}\right) \subset V$ is equivalent to $\operatorname{ker}\left(\tilde{g}-\mathbf{1}_{W}\right) \supset V$. When $g=\mathbf{1}_{V}$, the extensions $\tilde{g}$ are well known: see (Chevalley, 1954), section III.1.7; they are in bijection with the elements $\omega$ of $\wedge^{2}(V)$; if $\omega=\sum_{i=1}^{r} y_{i} \wedge z_{i}$, the associated orthogonal transformation $\mathrm{F}(\omega)$ maps each $(x, \ell)$ to $\left(x+\sum_{i}\left(\ell\left(y_{i}\right) z_{i}-\ell\left(z_{i}\right) y_{i}\right), \ell\right)$. Thus $\mathrm{F}(\omega) \circ \mathrm{F}\left(\omega^{\prime}\right)=\mathrm{F}\left(\omega+\omega^{\prime}\right)$. The calculation of the transformer $(S, \phi)$ associated with $\mathrm{F}(\omega)$ (according to Theorem 2.2 below) is easy when $\left(y_{1}, z_{1}, y_{2}, z_{2}, \ldots, y_{r}, z_{r}\right)$ is linearly independant: $S$ is the subspace with basis $\left(y_{1}, z_{1}, \ldots, y_{r}, z_{r}\right)$, and $\phi$ is the alternate bilinear form on $S$ such that $\phi\left(y_{i}, z_{i}\right)=1, \phi\left(y_{i}, z_{j}\right)=0$ if $i \neq j$, and $\phi\left(y_{i}, y_{j}\right)=\phi\left(z_{i}, z_{j}\right)=0$ for all $i$ and $j$. Thus we obtain a bijection between the elements of $\bigwedge^{2}(V)$ and the transformers $(S, \phi)$ of $(V, 0)$.

Let us suppose that the orthogonal transformation $g$ is a product of reflections $\mathrm{R}\left(v_{1}\right) \mathrm{R}\left(v_{2}\right) \cdots \mathrm{R}\left(v_{s}\right)$ involving $s$ linearly independent vectors; then $g$ admits the transformer $(S, \phi)$ where $S$ is the subspace with basis $\left(v_{1}, \ldots, v_{s}\right)$, and where $\phi$ has a lower triangular matrix in this basis; in other words, $\phi\left(v_{i}, v_{j}\right)=0$ whenever $i<j$; since $\phi(y, y)=q(y)$ for all $y \in S$, this property completely determines $\phi$. Conversely, if $(S, \phi)$ is a transformer for $g$, and if the matrix of $\phi$ is lower triangular in some basis $\left(v_{1}, \ldots, v_{s}\right)$ of $S$, then $g=\mathrm{R}\left(v_{1}\right) \cdots \mathrm{R}\left(v_{s}\right)$. Thus we are led to the problem which shall be the subject of the second part of this article: if $\phi$ is a bilinear form on a vector space $S$ (of finite dimension $s$ ), are there bases of $S$ where the matrix of $\phi$ is lower triangular, and how can we calculate one of them?

Although every transformer $(S, \phi)$ involves a non-degenerate bilinear form $\phi$, I will solve the problem of triangularization even when $\phi$ is degenerate; in the frame of Clifford algebras, there are at least two problems that require triangularisation even for degenerate bilinear forms. When $\phi$ is a non-zero alternate bilinear form, its matrix is alternate in every basis of $S$; therefore, it cannot be triangularized. All other bilinear forms can be triangularized, except when $K$ is isomorphic to $\mathbb{Z} / 2 \mathbb{Z}$. Bilinear forms over $\mathbb{Z} / 2 \mathbb{Z}$ are outside the scope of this article; here, I do not more than showing (just below) a bilinear form over $\mathbb{Z} / 2 \mathbb{Z}$ that cannot be triangularized although it is not alternate. I shall present an algorithm of triangularization where every phase is almost trivial, except the "correction procedure"; this procedure is the only phase that requires $K$ not to be isomorphic to $\mathbb{Z} / 2 \mathbb{Z}$; therefore, the presence of this unpleasant procedure is not the result of a clumsiness.

Example. Here, exceptionally, $K$ is the field $\mathbb{Z} / 2 \mathbb{Z}$. Let us consider the following non-degenerate bilinear form $\phi$ on $K^{3}$ :

$$
\phi\left(\left(\xi_{1}, \xi_{2}, \xi_{3}\right),\left(\zeta_{1}, \zeta_{2}, \zeta_{3}\right)\right)=\left(\xi_{1} \zeta_{2}-\xi_{2} \zeta_{1}\right)+\left(\xi_{2}+\xi_{3}\right) \zeta_{3} .
$$

If the matrix of $\phi$ is triangular in a basis $\left(v_{1}, v_{2}, v_{3}\right)$, then $\phi\left(v_{1}, v_{1}\right), \phi\left(v_{2}, v_{2}\right)$ and $\phi\left(v_{3}, v_{3}\right)$ are all $\neq 0$ because $\phi$ is nondegenerate. Unfortunately, only two vectors of $K^{3}$ are not isotropic for the quadratic form $v \longmapsto \phi(v, v)$ : $(0,0,1)$ and $(1,0,1)$. Therefore, $\phi$ cannot be triangularized.

\section{Preliminary Lemmas}

The first lemma is useful only in characteristic 2 .

Lemma 1.1. For every $g \in \operatorname{Iso}(V, q)$ we have $\operatorname{im}(g-1) \cap \operatorname{ker}\left(\mathrm{b}_{q}\right) \subset \operatorname{ker}(q)$; in other words, $\operatorname{im}(g-1) \cap \operatorname{ker}\left(\mathrm{b}_{q}\right)=$ $\operatorname{im}(g-\mathbf{1}) \cap \operatorname{ker}(q)$.

Proof. If $g(x)-x$ is in $\operatorname{ker}\left(\mathrm{b}_{q}\right)$, then

$$
q(x)=q(g(x))=q(x)+q(g(x)-x)+\mathrm{b}_{q}(x, g(x)-x)=q(x)+q(g(x)-x),
$$


whence $q(g(x)-x)=0$.

Lemma 1.1 implies that $\mathrm{O}(V, q)=\operatorname{Iso}(V, q)$ if and only if $\operatorname{ker}(q)=0$.

For every subspace $U$ of $V, U^{\perp}$ is the subspace of all $x \in V$ such that $\mathrm{b}_{q}(x, u)=0$ for all $u \in U$.

Lemma 1.2. For every $g \in \operatorname{Iso}(V, q)$, the subspaces $\operatorname{ker}(g-\mathbf{1})$ and $\operatorname{im}(g-\mathbf{1})$ are orthogonal. When $\operatorname{ker}(q)=0$, then $\operatorname{ker}(g-\mathbf{1})=(\operatorname{im}(g-\mathbf{1}))^{\perp}$.

Proof. For all $x, y \in V$ we have

$$
\mathrm{b}_{q}(x, g(y)-y)=-\mathrm{b}_{q}(g(x)-x, g(y)) ;
$$

therefore, every $x$ in $\operatorname{ker}(g-\mathbf{1})$ is orthogonal to every $g(y)-y \operatorname{in} \operatorname{im}(g-\mathbf{1})$. Conversely, if $x$ is orthogonal to all $g(y)-y$, then $g(x)-x$ is in $\operatorname{ker}\left(\mathrm{b}_{q}\right)$, therefore in $\operatorname{ker}(q)$; and $x \in \operatorname{ker}(g-\mathbf{1})$ if $\operatorname{ker}(q)=0$.

When $q$ is non-degenerate, the orthogonal group $\mathrm{O}(V, q)$ contains a normal subgroup $\mathrm{SO}(V, q)$ of index 2 which no reflection $\mathrm{R}(v)$ can belong to. The same holds true when $q$ is degenerate but non-defective; indeed, $q$ induces a non-degenerate quadratic form $q^{\prime \prime}$ on the quotient $V^{\prime \prime}=V / \operatorname{ker}(q)$, every $g \in \mathrm{O}(V, q)$ gives a transformation $g^{\prime \prime} \in \mathrm{O}\left(V^{\prime \prime}, q^{\prime \prime}\right)$, and $\operatorname{SO}(V, q)$ is the inverse image of $\mathrm{SO}\left(V^{\prime \prime}, q^{\prime \prime}\right)$ by the homomorphism $g \longmapsto g^{\prime \prime}$. If $g$ is a product of reflections, the parity of the number of reflections depends on whether $g$ is, or not, in the subgroup $\operatorname{SO}(V, q)$. All this is null and void when $q$ is defective; in this case, $\operatorname{ker}\left(\mathrm{b}_{q}\right)$ contains vectors $v$ such that $q(v) \neq 0$ and $\mathrm{R}(v)=\mathbf{1}_{V}$.

Now we consider a bilinear form $\phi$ on some vector space $S$, and we define the quadratic form $q$ by $q(y)=\phi(y, y)$ for all $y \in S$. Consequently,

$$
\forall x, y \in S, \quad \phi(x, y)+\phi(y, x)=\mathrm{b}_{q}(x, y) .
$$

Let $\operatorname{RKer}(\phi)(\operatorname{resp} . \operatorname{LKer}(\phi))$ be the subspace of all $x \in S$ such that $\phi(v, x)=0($ resp. $\phi(x, v)=0)$ for all $v \in S$. If $U$ is a subspace of $S$, we denote by $\mathrm{R}_{\phi}^{\perp}(U)$ (resp. $\left.\mathrm{L}_{\phi}^{\perp}(U)\right)$ the subspace of all $x \in S$ such that $\phi(u, x)=0$ (resp. $\left.\phi(x, u)=0\right)$ for all $u \in U$. When $U \subset \operatorname{ker}\left(\mathrm{b}_{q}\right)$, then $\mathrm{R}_{\phi}^{\perp}(U)=\mathrm{L}_{\phi}^{\perp}(U)$, and the notation $\operatorname{LR}_{\phi}^{\perp}(U)$ is allowed.

Lemma 1.3. Let $U_{1}$ and $U_{3}$ be two subspaces of $S$ such that $\phi\left(U_{1}, U_{3}\right)=0$ and such that the restrictions of $\phi$ to $U_{1}$ and $U_{3}$ are non-degenerate. Then we have $S=U_{1} \oplus U_{2} \oplus U_{3}$ if $U_{2}=\mathrm{R}_{\phi}^{\perp}\left(U_{1}\right) \cap \mathrm{L}_{\phi}^{\perp}\left(U_{3}\right)$.

Proof. For every $x \in S$, there is a unique $x_{1} \in U_{1}$ (resp. $x_{3} \in U_{3}$ ) such that $\phi(u, x)=\phi\left(u, x_{1}\right)$ for all $u \in U_{1}$ (resp. $\phi(x, u)=\phi\left(x_{3}, u\right)$ for all $\left.u \in U_{3}\right)$. If we set $p_{1}(x)=x_{1}$ and $p_{3}(x)=x_{3}$, then $p_{1}$ and $p_{3}$ are projectors such that $\operatorname{im}\left(p_{1}\right)=U_{1}, \operatorname{ker}\left(p_{1}\right)=\mathrm{R}_{\phi}^{\perp}\left(U_{1}\right), \operatorname{im}\left(p_{3}\right)=U_{3}, \operatorname{ker}\left(p_{3}\right)=\mathrm{L}_{\phi}^{\perp}\left(U_{3}\right)$. Since $\phi\left(U_{1}, U_{3}\right)=0$, we have $p_{1} p_{3}=p_{3} p_{1}=0$. Thus, if we set $p_{2}=\mathbf{1}-p_{1}-p_{3}$, we obtain a projector on $\operatorname{ker}\left(p_{1}\right) \cap \operatorname{ker}\left(p_{3}\right)=U_{2}$.

Lemma 1.3 can be applied when $U_{1}=0$ or $U_{3}=0$, because the unique bilinear form on $\{0\}$ is non-degenerate.

The next lemma, motivated by the frequent presence of $g-\mathbf{1}$, does not require $V$ to be a vector space; it holds true already for an additive group.

Lemma 1.4. Let $g_{1}$ and $g_{2}$ be homomorphisms from an additive group $V$ into itself, and $g=g_{1} g_{2}$ their product. Let us consider these four assertions:

$$
\begin{aligned}
(\text { im }): & & \operatorname{im}\left(g_{1}-\mathbf{1}\right) \cap \operatorname{im}\left(g_{2}-\mathbf{1}\right) & =0 ; \\
(\text { Im }): & & \operatorname{im}\left(g_{1}-\mathbf{1}\right)+\operatorname{im}\left(g_{2}-\mathbf{1}\right) & =\operatorname{im}(g-\mathbf{1}) ; \\
(\text { ker }): & & \operatorname{ker}\left(g_{1}-\mathbf{1}\right)+\operatorname{ker}\left(g_{2}-\mathbf{1}\right) & =V ; \\
(\text { Ker }): & & \operatorname{ker}\left(g_{1}-\mathbf{1}\right) \cap \operatorname{ker}\left(g_{2}-\mathbf{1}\right) & =\operatorname{ker}(g-\mathbf{1}) .
\end{aligned}
$$

The following four implications hold true:

$$
\begin{aligned}
(\mathrm{im}) \Rightarrow(\mathrm{Ker}), & (\mathrm{ker}) \Rightarrow(\mathrm{Im}) ; \\
(\mathrm{im}) \&(\mathrm{Im}) & \Longleftrightarrow(\mathrm{ker}) \&(\mathrm{Ker}) .
\end{aligned}
$$

Proof. I will prove only (1.2) because we shall never use (1.3) which is mentioned here only because it would be a pity to mutilate Lemma 1.4; yet the proof of (1.3) is more difficult. The two inclusions

$$
\operatorname{im}\left(g_{1}-\mathbf{1}\right)+\operatorname{im}\left(g_{2}-\mathbf{1}\right) \supset \operatorname{im}(g-\mathbf{1}) \quad \text { and } \quad \operatorname{ker}\left(g_{1}-\mathbf{1}\right) \cap \operatorname{ker}\left(g_{2}-\mathbf{1}\right) \subset \operatorname{ker}(g-\mathbf{1})
$$

are obvious consequences of

$$
g-\mathbf{1}=\left(g_{1}-\mathbf{1}\right) g_{2}+\left(g_{2}-\mathbf{1}\right)=g_{1}\left(g_{2}-\mathbf{1}\right)+\left(g_{1}-\mathbf{1}\right) .
$$


Let us prove $(\mathrm{im}) \Rightarrow(\mathrm{Ker})$. If $(\mathrm{im})$ is true and $g(x)=x$, then $\left(g_{1}-\mathbf{1}\right) g_{2}(x)=\left(g_{2}-\mathbf{1}\right)(x)=0$, whence $g_{2}(x)=x=g_{1}(x)$; this means that $(K e r)$ is true. Now let us prove that $(k e r) \operatorname{implies} \operatorname{im}\left(g_{1}-\mathbf{1}\right) \subset \operatorname{im}(g-\mathbf{1})$; $\operatorname{since} \operatorname{im}\left(g_{2}-\mathbf{1}\right) \subset \operatorname{im}(g-\mathbf{1})$ for the same reasons, (Im) follows. Let us consider $y=\left(g_{1}-\mathbf{1}\right)(x)$ and let us write $x=x_{1}+x_{2}$ where $g_{1}\left(x_{1}\right)=x_{1}$ and $g_{2}\left(x_{2}\right)=x_{2} ;$ thus $y=\left(g_{1}-\mathbf{1}\right)\left(x_{2}\right)=g_{1}\left(g_{2}-\mathbf{1}\right)\left(x_{2}\right)+\left(g_{1}-\mathbf{1}\right)\left(x_{2}\right)=(g-\mathbf{1})\left(x_{2}\right)$.

Remark. When $\operatorname{dim}(V)$ is infinite, which properties of an isometry $g$ ensure that it extends to a twisted inner automorphism of $\mathrm{Cl}(V, q)$ ? The necessary condition $\operatorname{ker}(g-\mathbf{1}) \supset \operatorname{ker}\left(\mathrm{b}_{q}\right)$ is no longer sufficient. Indeed, an isometry $g$ extends to a twisted inner automorphism (and is called an orthogonal transformation) if and only if the codimension of $\operatorname{ker}(g-\mathbf{1})$ is finite, and if $\operatorname{ker}(g-\mathbf{1})$ is orthogonally closed according to this definition: a subspace $U$ of $V$ is orthogonally closed if $U^{\perp \perp}=U$. I recall that $U^{\perp \perp} \supset U$ and $U^{\perp \perp \perp}=U^{\perp}$ for every subspace $U$. When the codimension of $\operatorname{ker}\left(\mathrm{b}_{q}\right)$ is infinite, the property $\operatorname{ker}(g-\mathbf{1}) \supset \operatorname{ker}\left(\mathrm{b}_{q}\right)$ is much weaker. When $\operatorname{ker}(q)=0$, then $\operatorname{ker}(g-\mathbf{1})$ is orthogonally closed for every isometry $g$ because Lemma 1.2 is always valid. But if $\operatorname{ker}(q)$ contains a vector $u \neq 0$, then every $\ell \in V^{*}$ determines an isometry $g: x \longmapsto x+\ell(x) u$ such that $\operatorname{ker}(g-\mathbf{1})=\operatorname{ker}(\ell)$; and $g$ is an orthogonal transformation if and only if there is $v \in V$ such that $\ell(x)=\mathrm{b}_{q}(v, x)$ for all $x \in V$; even when $\operatorname{ker}(\ell) \supset \operatorname{ker}\left(\mathrm{b}_{q}\right)$, the existence of $v$ is exceptional. Besides, for every orthogonal transformation $g$, there is an orthogonal decompostion $V=V_{1} \oplus V_{2}$ such that $\operatorname{dim}\left(V_{1}\right)$ is finite, $\operatorname{im}(g-\mathbf{1}) \subset V_{1}$ and $\operatorname{ker}(g-\mathbf{1}) \supset V_{2}$; it reduces the study of $g$ to the finite-dimensional case. Nothing interesting will occur as long as no other concept and no other hypothesis (for instance, the presence of a topology) is introduced.

\section{The Main Theorems for Transformers}

A transformer of $(V, q)$ is a couple $(S, \phi)$ where $\phi$ is a non-degenerate bilinear form on a subspace $S$ of $V$, and satisfies the condition $\phi(y, y)=q(y)$ for all $y \in S$. The following two theorems justify this definition.

Theorem 2.1. Let $(S, \phi)$ be a transformer of $(V, q)$. There is a unique linear endomorphism $g$ of $V$ such that $\operatorname{im}(g-\mathbf{1}) \subset S$, and such that

$$
\forall x \in V, \forall y \in S, \quad \phi(g(x)-x, y)=-\mathrm{b}_{q}(x, y) ;
$$

it is an orthogonal transformation of $(V, q)$. Moreover,

$$
\begin{aligned}
\operatorname{ker}(g-\mathbf{1}) & =S^{\perp}, \\
\operatorname{im}(g-\mathbf{1}) & =\operatorname{LR}_{\phi}^{\perp}\left(S \cap \operatorname{ker}\left(\mathrm{b}_{q}\right)\right) ; \\
\operatorname{dim}(S) & \geq \operatorname{dim}(\operatorname{im}(g-\mathbf{1}))+\operatorname{dim}(\operatorname{im}(g-\mathbf{1}) \cap \operatorname{ker}(q)) ; \\
\forall y, z \in S, \quad \phi(g(y), g(z)) & =\phi(y, z) .
\end{aligned}
$$

The reverse transformer $\left(S, \phi^{\dagger}\right)$, where $\phi^{\dagger}$ is defined by $\phi^{\dagger}(x, y)=\phi(y, x)$, gives the inverse transformation $g^{-1}$.

Proof. Since $\phi$ is non-degenerate, it is clear that (2.1) determines an endomorphism $g$. Every $x \in \operatorname{ker}(g)$ must be in $S$, and $\phi(x, y)=\mathrm{b}_{q}(x, y)$ for all $y \in S$, whence $\phi(y, x)=0$ because of (1.1), and $x=0$ since $\phi$ is non-degenerate. Therefore, $g$ is bijective. Let us prove that it is an isometry; for all $x \in V$, we have $g(x)=x+(g(x)-x)$, whence

$$
\begin{aligned}
q(g(x))-q(x) & =q(g(x)-x)+\mathrm{b}_{q}(x, g(x)-x) \\
& =q(g(x)-x)-\phi(g(x)-x, g(x)-x)=q(y)-\phi(y, y) \quad \text { if } y=g(x)-x ;
\end{aligned}
$$

thus $q(g(x))=q(x)$ as expected. From (2.1) we deduce that $g(x)-x=0$ if and only if $x \in S^{\perp}$; consequently, (2.2) holds true, and $g$ is an orthogonal transformation. If $\ell$ is a linear form on $S$, there is $x \in V$ such that $\ell(y)=-\mathrm{b}_{q}(x, y)$ for all $y \in S$ if and only if $\ell$ vanishes on $S \cap \operatorname{ker}\left(\mathrm{b}_{q}\right)$. On another side, a vector $z$ of $S$ belongs to $\operatorname{im}(g-\mathbf{1})$ if and only if the linear form $y \longmapsto \phi(z, y)$ is equal to $y \longmapsto-\mathrm{b}_{q}(x, y)$ for some $x \in V$; this occurs if and only if $z \in \mathrm{L}_{\phi}^{\perp}\left(S \cap \operatorname{ker}\left(\mathrm{b}_{q}\right)\right)$; this proves (2.3). Since $\phi$ is non-degenerate,

$$
\begin{aligned}
\operatorname{dim}(S) & =\operatorname{dim}\left(S \cap \operatorname{ker}\left(\mathrm{b}_{q}\right)\right)+\operatorname{dim}\left(\mathrm{L}_{\phi}^{\perp}\left(S \cap \operatorname{ker}\left(\mathrm{b}_{q}\right)\right)\right) \\
& \geq \operatorname{dim}(\operatorname{im}(g-\mathbf{1}) \cap \operatorname{ker}(q))+\operatorname{dim}(\operatorname{im}(g-\mathbf{1})),
\end{aligned}
$$

in accordance with (2.4). The fact that $g^{-1}$ can be derived from $\left(S, \phi^{\dagger}\right)$ is equivalent to the following fact:

$$
\forall y \in S, \forall x \in V, \quad \phi(y, g(x)-x)=\mathrm{b}_{q}(y, g(x)) ;
$$

this formula (2.7) is a consequence of (1.1) and (2.1):

$$
\phi(y, g(x)-x)=\mathrm{b}_{q}(g(x)-x, y)-\phi(g(x)-x, y)=\mathrm{b}_{q}(g(x)-x, y)+\mathrm{b}_{q}(x, y)=\mathrm{b}_{q}(g(x), y) .
$$

Finally, we derive (2.5) from (2.1) and (2.6); for all $y, z \in S$,

$$
\phi(g(y), g(z))-\phi(y, z)=\phi(g(y)-y, g(z))+\phi(y, g(z)-z)=-\mathrm{b}_{q}(y, g(z))+\mathrm{b}_{q}(y, g(z))=0 .
$$


The proof of Theorem 2.1 is complete.

When $q$ is non-degenerate, the equality (2.3) means that $\operatorname{im}(g-\mathbf{1})=S$. A transformer $(S, \phi)$ gives the transformation $\mathbf{1}$ if and only if $S \subset \operatorname{ker}\left(\mathrm{b}_{q}\right)$. The trivial transformer $(0,0)$ (on the null subspace $\{0\}$ ) always gives $\mathbf{1}$. Now we come to the reciprocal theorem.

Theorem 2.2. Every $g \in \mathrm{O}(V, q)$ admits a transformer $(S, \phi)$ such that

$$
\operatorname{dim}(S)=\operatorname{dim}(\operatorname{im}(g-\mathbf{1}))+\operatorname{dim}(\operatorname{im}(g-\mathbf{1}) \cap \operatorname{ker}(q)) .
$$

We can require $S$ not to be totally isotropic, except in these two cases:

$$
\begin{aligned}
& \text { if } \operatorname{im}(g-\mathbf{1}) \cap \operatorname{ker}(q)=0 \text { and } \operatorname{im}(g-\mathbf{1}) \text { is totally isotropic; } \\
& \text { if } \operatorname{im}(g-\mathbf{1}) \cap \operatorname{ker}(q) \neq 0 \text { and }(\operatorname{ker}(g-\mathbf{1}))^{\perp} \text { is totally isotropic. }
\end{aligned}
$$

Proof. There is an easy case and a difficult case.

The easy case: $\operatorname{im}(g-\mathbf{1}) \cap \operatorname{ker}(q)=0$. In this case, (2.7) means that $S=\operatorname{im}(g-\mathbf{1})$. Let us prove that the equation (2.1) determines a bilinear form $\phi$; we must verify that every equality $g(x)-x=g\left(x^{\prime}\right)-x^{\prime}$ implies $\mathrm{b}_{q}(x, y)=\mathrm{b}_{q}\left(x^{\prime}, y\right)$ for all $y \in S$; indeed, this equality means $x-x^{\prime} \in \operatorname{ker}(g-\mathbf{1})$; therefore, $x-x^{\prime}$ is orthogonal to $\operatorname{im}(g-\mathbf{1})=S$ and $\mathrm{b}_{q}\left(x-x^{\prime}, y\right)=0$. This bilinear form $\phi$ is non-degenerate; indeed, if $\phi(z, y)=0$ for all $z \in S$, then $\mathrm{b}_{q}(x, y)=0$ for all $x \in V$, therefore $y \in \operatorname{ker}\left(\mathrm{b}_{q}\right)$, whence $y \in S \cap \operatorname{ker}\left(\mathrm{b}_{q}\right)=\operatorname{im}(g-\mathbf{1}) \cap \operatorname{ker}(q)=0$. When $y=g(x)-x$, we can prove that $q(g(x))-q(x)=q(y)-\phi(y, y)$ as we did it in the proof of Theorem 2.1; and here, this equality implies $\phi(y, y)=q(y)$ for all $y \in S$.

The difficult case: $\operatorname{im}(g-\mathbf{1}) \cap \operatorname{ker}(q) \neq 0$. Let $\left(b_{1}, \ldots, b_{t}\right)$ be a basis of $S_{0}=\operatorname{im}(g-\mathbf{1}) \cap \operatorname{ker}(q)$, and $S^{\prime}$ a subspace such that $\operatorname{im}(g-\mathbf{1})=S_{0} \oplus S^{\prime}$. Moreover, let $V^{\prime}$ be a subspace such that $V=\operatorname{ker}\left(\mathrm{b}_{q}\right) \oplus V^{\prime}$ and $V^{\prime} \supset S^{\prime}$. Since $q$ is non-degenerate on $V^{\prime}$, there is an orthogonal transformation $g^{\prime}$ of $V^{\prime}$ and there is $\left(c_{1}, \ldots, c_{t}\right)$ in $V^{\prime}$ such that

$$
\forall x \in V^{\prime}, \quad g(x)=g^{\prime}(x)+\sum_{i=1}^{t} \mathrm{~b}_{q}\left(x, c_{i}\right) b_{i} .
$$

In $V^{\prime}$ we can find a linearly independent sequence $\left(a_{1}, \ldots, a_{t}\right)$ such that $g\left(a_{i}\right)-a_{i}=b_{i}$ for $i=1,2, \ldots, t$. Consequently, $g^{\prime}\left(a_{i}\right)=a_{i}$ and $\mathrm{b}_{q}\left(a_{i}, c_{i}\right)=1$ for $i=1,2, \ldots, t$, but $\mathrm{b}_{q}\left(a_{i}, c_{j}\right)=0$ if $i \neq j$. This proves that $\left(c_{1}, \ldots, c_{t}\right)$ spans a subspace $S_{1}$ of dimension $t$ which $\mathrm{b}_{q}$ puts in duality with the space spanned by $\left(a_{1}, \ldots, a_{t}\right)$. Moreover, $S_{1} \cap\left(S_{0} \oplus S^{\prime}\right)=0$ because $S_{0} \oplus S^{\prime}$ (that is $\left.\operatorname{im}(g-\mathbf{1})\right)$ is orthogonal to the subspace spanned by $\left(a_{1}, \ldots, a_{t}\right)$; indeed, for all $x \in V$,

$$
\mathrm{b}_{q}\left(a_{i}, g(x)-x\right)=-\mathrm{b}_{q}\left(g\left(a_{i}\right)-a_{i}, g(x)\right)=-\mathrm{b}_{q}\left(b_{i}, g(x)\right)=0 .
$$

Let us set $S=S_{0} \oplus S^{\prime} \oplus S_{1}$. This subspace $S$ is orthogonal to $\operatorname{ker}(g-\mathbf{1})$; indeed, we already know that $S_{0} \oplus S^{\prime}$ (that is $\operatorname{im}(g-\mathbf{1}))$ is orthogonal to $\operatorname{ker}(g-\mathbf{1})$; $\operatorname{since} \operatorname{ker}(g-\mathbf{1}) \supset \operatorname{ker}\left(\mathrm{b}_{q}\right)$, it suffices to prove that $S_{1}$ is orthogonal to $V^{\prime} \cap \operatorname{ker}(g-\mathbf{1})$; this follows from (12.8), where the equality $g(x)=x$ implies implies $\mathrm{b}_{q}\left(x, c_{i}\right)=0$ for $i=1,2, \ldots, t$.

Now we construct $\phi$. The equation (2.1) involves only the restriction of $\phi$ to $\left(S_{0} \oplus S^{\prime}\right) \times S$, and as in the previous easy case, it actually determines this restriction, because every equality $g(x)-x=g\left(x^{\prime}\right)-x^{\prime}$ implies that $x-x^{\prime}$ is in ker $(g-\mathbf{1})$, therefore orthogonal to $S$. Since $S_{0} \subset \operatorname{ker}\left(\mathrm{b}_{q}\right)$, it is clear that $\phi$ vanishes on $\left(S_{0} \oplus S^{\prime}\right) \times S_{0}$. Since the vectors $a_{i}$ are orthogonal to $S_{0} \oplus S^{\prime}$ (see above), $\phi$ vanishes on $S_{0} \times\left(S_{0} \oplus S^{\prime}\right)$ too:

$$
\phi\left(b_{i}, y\right)=\phi\left(g\left(a_{i}\right)-a_{i}, y\right)=-\mathrm{b}_{q}\left(a_{i}, y\right)=0 \quad \text { if } y \in S_{0} \oplus S^{\prime} .
$$

Since $\phi\left(b_{i}, c_{j}\right)=\phi\left(g\left(a_{i}\right)-a_{i}, c_{j}\right)=-\mathrm{b}_{q}\left(a_{i}, c_{j}\right)$, we have $\phi\left(b_{i}, c_{i}\right)=-1$, but $\phi\left(b_{i}, c_{j}\right)=0$ if $i \neq j$. On another side, the restriction of $\phi$ to $S^{\prime}$ is non-degenerate; indeed, if $y$ is an element of $S^{\prime}$ such that $\phi(z, y)=0$ for all $z \in S^{\prime}$, then $\phi(z, y)=0$ for all $z \in S_{0} \oplus S^{\prime}$; therefore, $\mathrm{b}_{q}(x, y)=-\phi(g(x)-x, y)=0$ for all $x \in V$, whence $y \in S^{\prime} \cap \operatorname{ker}\left(\mathrm{b}_{q}\right)=0$. Since the equation (2.1) is now satisfied, we can deduce the equality $q(g(x))-q(x)=q(y)-\phi(y, y)$ from $y=g(x)-x$ as above, and claim that $\phi(y, y)=q(y)$ for all $y \in S_{0} \oplus S^{\prime}$. To complete the construction of $\phi$, we have only to worry about the equalities $\phi(y, y)=q(y)$ and $\phi(y, z)+\phi(z, y)=\mathrm{b}_{q}(y, z)$ when $y$ is in $S_{1}$. Since $S_{0}$ and $S_{1}$ are orthogonal, we realize that $\phi\left(c_{i}, b_{i}\right)=1$ for $i=1,2, \ldots, t$, but $\phi\left(c_{i}, b_{j}\right)=0$ if $i \neq j$. Let us choose a basis $\left(d_{1}, \ldots, d_{r}\right)$ of $S^{\prime}$, and consider the matrix $\Phi$ of $\phi$ in the basis $\left(b_{1}, \ldots, b_{t}, d_{1}, \ldots, d_{r}, c_{1}, \ldots, c_{t}\right)$ of $S$ :

$$
\Phi=\left(\begin{array}{ccc}
0 & 0 & -\mathbf{1}_{t} \\
0 & M & N \\
\mathbf{1}_{t} & N^{\prime} & P
\end{array}\right)
$$


the submatrix $M$ is invertible since it gives the restriction of $\phi$ to $S^{\prime}$; consequently the matrix $\Phi$ is invertible. The submatrix $N^{\prime}$ is determined by $N$ and the restriction of $\mathrm{b}_{q}$ to $S_{1} \times S^{\prime}$; but when $t \geq 2$, the submatrix $P$ is not completely determined by the condition $\phi(y, y)=q(y)$ for all $y \in S_{1}$.

It remains to prove that there are non totally isotropic choices of $S$ if and only if $\operatorname{ker}(g-\mathbf{1})^{\perp}$ is not totally isotropic. When $q$ is defective, there is $u \in \operatorname{ker}\left(\mathrm{b}_{q}\right)$ such that $q(u) \neq 0$; since $\operatorname{ker}(g-\mathbf{1})^{\perp}$ contains $u$, it is never totally isotropic, and we must prove that there is always a non totally isotropic choice of $S$; indeed, the equality (2.8) remains true if we replace $c_{1}$ with $c_{1}+u$; since $q\left(c_{1}+u\right)=q\left(c_{1}\right)+q(u) \neq q\left(c_{1}\right)$, we can choose $c_{1}$ in such a way that $q\left(c_{1}\right) \neq 0$. Now let us suppose that $\operatorname{ker}(q)=\operatorname{ker}\left(\mathrm{b}_{q}\right)$. Since (2.2) implies $S \subset \operatorname{ker}(g-\mathbf{1})^{\perp}$, every choice of $S$ is totally isotropic if $\operatorname{ker}(g-\mathbf{1})^{\perp}$ is totally isotropic. Conversely, let the above constructed subspace $S$ be totally isotropic, and let us prove that $V^{\prime} \cap \operatorname{ker}(g-\mathbf{1})^{\perp}$ is totally isotropic (therefore, $\operatorname{ker}(g-\mathbf{1})^{\perp}$ too). From (2.8) we deduce that $V^{\prime} \cap \operatorname{ker}(g-\mathbf{1})$ is the intersection of $V^{\prime} \cap S_{1}^{\perp}$ and $\operatorname{ker}\left(g^{\prime}-\mathbf{1}_{V^{\prime}}\right)$, and also that $\operatorname{im}\left(g^{\prime}-\mathbf{1}_{V^{\prime}}^{\prime}\right)=S^{\prime}$. Since $q$ is non-degenerate on $V^{\prime}, \operatorname{ker}\left(g^{\prime}-\mathbf{1}_{V^{\prime}}\right)=V^{\prime} \cap S^{\prime \perp}$. Thus $V^{\prime} \cap \operatorname{ker}(g-\mathbf{1})$ is the intersection of $V^{\prime} \cap S^{\prime \perp}$ and $V^{\prime} \cap S_{1}^{\perp}$, whence $V^{\prime} \cap \operatorname{ker}(g-\mathbf{1})^{\perp}=S^{\prime} \oplus S_{1}$. If $S$ is totally isotropic, the same is true for $S^{\prime} \oplus S_{1}$ and $\operatorname{ker}(g-\mathbf{1})^{\perp}$.

When $q$ is non-degenerate, the correspondance between transformers and orthogonal transformations is bijective. In Section 4, it is explained that the same is true for a non-defective $q$ such that $\operatorname{dim}(\operatorname{ker}(q))=1$. Whatever $q$ may be, if $(V, q) \rightarrow(W, \tilde{q})$ is an embedding such that $V \subset W$, every transformer $(S, \phi)$ of $(V, q)$ is also a transformer of $(W, \tilde{q})$; consequently, every $g \in \mathrm{O}(V, q)$ has an extension $\tilde{g} \in \mathrm{O}(W, \tilde{q})$ such that $\operatorname{im}\left(\tilde{g}-\mathbf{1}_{W}\right) \subset V$. Conversely, if $\tilde{q}$ is non-degenerate, every $\tilde{g} \in \mathrm{O}(W, \tilde{q})$ such that $\operatorname{im}\left(\tilde{g}-\mathbf{1}_{W}\right) \subset V$ admits a transformer $(S, \phi)$ such that $S \subset V$; thus there is a bijection between the transformers of $(V, q)$ and the elements $\tilde{g} \in \mathrm{O}(W, \tilde{q})$ such that $\operatorname{im}\left(\tilde{g}-\mathbf{1}_{W}\right) \subset V$. This fact gives a structure of group on the set of transformers of $(V, q)$. This structure does not depend on the choice of the embedding; indeed, if $(V, q)$ is embedded in $(W, \tilde{q})$ and in $\left(W^{\prime}, \tilde{q}^{\prime}\right)$ (with non-degenerate $\tilde{q}$ and $\left.\tilde{q}^{\prime}\right)$, then $(W, \tilde{q})$ and $\left(W^{\prime}, \tilde{q}^{\prime}\right)$ can be embedded in the same non-degenerate space $\left(W^{\prime \prime}, \tilde{q}^{\prime \prime}\right)$ in such a way that we get twice the same embedding $(V, q) \rightarrow\left(W^{\prime \prime}, \tilde{q}^{\prime \prime}\right)$; it is easy to construct $\left(W^{\prime \prime}, \tilde{q}^{\prime \prime}\right)$ (despite a little difficulty when $q$ is defective).

When $K$ is the field $\mathbb{R}$ of real numbers, the groups under consideration are Lie groups. The dimension of the group of transformers is always $n(n-1) / 2$; indeed, there is canonical bijection from $\bigwedge^{2}(W)$ onto the Lie algebra of $\mathrm{O}(W, \tilde{q})$ which maps every $y \wedge z$ to the operator $x \longmapsto \mathrm{b}_{\tilde{q}}(x, y) z-\mathrm{b}_{\tilde{q}}(x, z) y$, and the image of $\wedge^{2}(V)$ is actually the Lie algebra of the subgroup determined by the condition $\operatorname{im}\left(\tilde{g}-\mathbf{1}_{W}\right) \subset V$. The dimension of $\mathrm{O}(V, q)$ depends on $k=\operatorname{dim}(\operatorname{ker}(q))$; it is $(n(n-1)-k(k-1)) / 2=(n-k)(n+k-1) / 2$. The group $\operatorname{Iso}(V, q)$ is isomorphic to a semi-direct product of $\mathrm{O}(V, q)$ and $\operatorname{GL}(\operatorname{ker}(q))$.

Theorem 2.3 gives an example of a product of transformers.

Theorem 2.3. Let $\left(S_{1}, \phi_{1}\right)$ and $\left(S_{2}, \phi_{2}\right)$ be two transformers of $(V, q)$ such that $S_{1} \cap S_{2}=0$, and let $g_{1}$ and $g_{2}$ be the associated orthogonal transformations. Their product $g=g_{1} g_{2}$ admits the following transformer $(S, \phi): S=S_{1} \oplus S_{2}$; $\phi$ coincides with $\phi_{1}$ on $S_{1}$, with $\phi_{2}$ on $S_{2}$, and for all $y_{1} \in S_{1}$ and $y_{2} \in S_{2}$ we have $\phi\left(y_{1}, y_{2}\right)=0\left(\right.$ whence $\phi\left(y_{2}, y_{1}\right)=$ $\left.\mathrm{b}_{q}\left(y_{1}, y_{2}\right)\right)$.

Proof. Since $(V, q)$ can be embedded in a non-degenerate space $(W, \tilde{q})$, it suffices to prove Theorem 2.3 when $q$ is nondegenerate. This hypothesis implies $\operatorname{im}\left(g_{1}-\mathbf{1}\right)=S_{1}$ and $\operatorname{ker}\left(g_{1}-\mathbf{1}\right)=S_{1}^{\perp}$, and similarly $\operatorname{im}\left(g_{2}-\mathbf{1}\right)=S_{2}$ and $\operatorname{ker}\left(g_{2}-\mathbf{1}\right)=$ $S_{2}^{\perp}$. Since $S_{1} \cap S_{2}=0$, we have $S_{1}^{\perp}+S_{2}^{\perp}=V$, consequently, $\operatorname{ker}\left(g_{1}-\mathbf{1}\right)+\operatorname{ker}\left(g_{2}-\mathbf{1}\right)=V$, and Lemma 1.4 implies that $\operatorname{im}(g-\mathbf{1})=\operatorname{im}\left(g_{1}-\mathbf{1}\right)+\operatorname{im}\left(g_{2}-\mathbf{1}\right)$. It follows that $S=S_{1} \oplus S_{2}$.

Let us consider vectors $x, y_{1}$ and $y_{2}$ respectively in $V, S_{1}$ and $S_{2}$. Let us calculate $\phi\left(g(x)-x, y_{2}\right)$ when $g(x)-x$ is in $S_{2}$; from $g-\mathbf{1}=\left(g_{1}-\mathbf{1}\right) g_{2}+\left(g_{2}-\mathbf{1}\right)$ and $S_{1} \cap S_{2}=0$, we deduce $g(x)-x=g_{2}(x)-x$; consequently,

$$
\phi\left(g(x)-x, y_{2}\right)=-\mathrm{b}_{q}\left(x, y_{2}\right)=\phi_{2}\left(g_{2}(x)-x, y_{2}\right)=\phi_{2}\left(g(x)-x, y_{2}\right) ;
$$

therefore, $\phi$ coincides with $\phi_{2}$ on $S_{2}$. Now we suppose that $g(x)-x$ is in $S_{1}$; for the same reasons as above, this implies $g_{2}(x)=x$ and $g(x)-x=g_{1}(x)-x$; consequently,

$$
\begin{aligned}
& \phi\left(g(x)-x, y_{1}\right)=-\mathrm{b}_{q}\left(x, y_{1}\right)=\phi_{1}\left(g_{1}(x)-x, y_{1}\right)=\phi_{1}\left(g(x)-x, y_{1}\right), \\
& \phi\left(g(x)-x, y_{2}\right)=-\mathrm{b}_{q}\left(x, y_{2}\right)=\phi_{2}\left(g_{2}(x)-x, y_{2}\right)=0 ;
\end{aligned}
$$

therefore, $\phi$ coincides with $\phi_{1}$ on $S_{1}$, and $\phi\left(S_{1}, S_{2}\right)=0$.

Corollary 2.4. Let $\left(S_{1}, \phi_{1}\right)$ and $\left(S_{2}, \phi_{2}\right)$ be two transformers of $(V, q)$ such that $S_{1} \subset S_{2}$, and $\phi_{1}(y, z)=\phi_{2}(z, y)$ for all $y, z \in S_{1}$. Let $g_{1}$ and $g_{2}$ be the associated orthogonal transformations. Their product $g=g_{1} g_{2}$ admits the following transformer $(S, \phi): S=\mathrm{R}_{\phi_{2}}^{\perp}\left(S_{1}\right)$ and $\phi$ is the restriction of $\phi_{2}$ to $S$. And their product $g^{\prime}=g_{2} g_{1}$ admits the following transformer $\left(S^{\prime}, \phi^{\prime}\right): S^{\prime}=\mathrm{L}_{\phi_{2}}^{\perp}\left(S_{1}\right)$ and $\phi^{\prime}$ is the restriction of $\phi_{2}$ to $S^{\prime}$. 
Proof. The equalities $g=g_{1} g_{2}$ and $g^{\prime}=g_{2} g_{1}$ are equivalent to $g_{2}=g_{1}^{-1} g$ and $g_{2}=g^{\prime} g_{1}^{-1}$, and $g_{1}^{-1}$ is given by the reverse transformer $\left(S_{1}, \phi_{1}^{\dagger}\right)$ where $\phi_{1}^{\dagger}$ coincides with the restriction of $\phi_{2}$ to $S_{1}$. Since $\phi_{1}$ is non-degenerate, we have $S_{2}=S_{1} \oplus \mathrm{R}_{\phi_{2}}^{\perp}\left(S_{1}\right)$ and $S_{2}=\mathrm{L}_{\phi_{2}}^{\perp}\left(S_{1}\right) \oplus S_{1}$ (see Lemma 1.3). With Theorem 2.3, it is easy to verify that $g_{2}=g_{1}^{-1} g$ and $g_{2}=g^{\prime} g_{1}^{-1}$ if $g$ and $g^{\prime}$ are determined by the transformers described in Corollary 2.4.

\section{Products of Reflections}

Let $(S, \phi)$ be a transformer of $(V, q)$ such that $\operatorname{dim}(S)=1$; thus $S$ is spanned by a non-zero vector $v$ and $\phi(v, v)=q(v)$; since $\phi$ is non-degenerate, we have $q(v) \neq 0$ and $v$ determines a reflection $\mathrm{R}(v)$; and since $\phi(\mathrm{R}(v)(x)-x, v)=-\mathrm{b}_{q}(x, v)$ for all $x \in V$, we realize that $\mathrm{R}(v)$ admits $(S, \phi)$ as a transformer. Thus the reflections are the orthogonal transformations determined by the one-dimensional transformers. The following theorem is an immediate consequence of Theorem 2.3 and Corollary 2.4 .

Theorem 3.1. Let us consider a reflection $\mathrm{R}(v)$ and the orthogonal transformation $h$ determined by a transformer $(T, \psi)$. The products $g=\mathrm{R}(v) h$ and $g^{\prime}=h \mathrm{R}(v)$ admit the following transformers $(S, \phi)$ and $\left(S^{\prime}, \phi^{\prime}\right)$ :

if $v$ is outside $T$, then $S=S^{\prime}=T \oplus K v$, the restrictions of $\phi$ and $\phi^{\prime}$ to $T$ coincide with $\psi$, and $\phi(v, y)=\phi^{\prime}(y, v)=0$ for all $y \in T$ (whence $\phi(y, v)=\phi^{\prime}(v, y)=\mathrm{b}_{q}(v, y)$; and of course, $\phi(v, v)=\phi^{\prime}(v, v)=q(v)$ );

if $v$ belongs to $T$, then $S=\mathrm{R}_{\psi}^{\perp}(v)$ and $S^{\prime}=\mathrm{L}_{\psi}^{\perp}(v)$, and $\phi$ and $\phi^{\prime}$ are the restrictions of $\psi$ to $S$ and $S^{\prime}$ respectively.

Corollary 3.2. For every $g \in \mathrm{O}(V, q)$ and for every sequence $\left(v_{1}, v_{2}, \ldots, v_{s}\right)$ of linearly independent vectors in $V$, these two assertions are equivalent:

$g=\mathrm{R}\left(v_{1}\right) \mathrm{R}\left(v_{2}\right) \cdots \mathrm{R}\left(v_{s}\right) ;$

$g$ admits the transformer $(S, \phi)$ where $\left(v_{1}, \ldots, v_{s}\right)$ is a basis of $S$, and $\phi$ has a lower triangular matrix in this basis.

Theorem 3.1 and its corollary provide an effective method to calculate the product $(S, \phi)$ of two transformers $\left(S_{1}, \phi_{1}\right)$ and $\left(S_{2}, \phi_{2}\right)$ when a triangularizing basis is known for one factor. Since $S \subset S_{1}+S_{2}$, the product can be calculated in the subspace $S_{1}+S_{2}$ without worrying about the non-degenerate embeddings that were previously necessary to prove that it is well defined. For instance, if $(S, \phi)$ is the transformer for a product of reflections $\mathrm{R}\left(w_{1}\right) \cdots \mathrm{R}\left(w_{k}\right)$, then $S$ is contained in the subspace spanned by $\left(w_{1}, \ldots, w_{k}\right)$.

Section 5 shall be devoted to the proof of the next theorem, and to the construction of an effective algorithm of triangularization; this theorem requires the hypotheses that $K$ is not isomorphic to $\mathbb{Z} / 2 \mathbb{Z}$.

Theorem 3.3. If $\phi$ is a bilinear form on some space $S$, and if $\phi$ is not alternate, there are bases of $S$ where the matrix of $\phi$ is lower triangular.

In Theorem 3.3, it is clear that $\phi$ is alternate if and only if $S$ is totally isotropic for the quadratic form $y \longmapsto \phi(y, y)$.

The previous statements enable us to prove that every $g \in \mathrm{O}(V, q)$ can be decomposed into a product of reflections, and to evaluate the minimal number of reflections in such a decomposition. The minimal dimension of a transformer for $g$ is given by (2.7); as in the proof of Theorem 2.2, we consider two cases (and we suppose $g \neq \mathbf{1}_{V}$ ).

In the easy case $\operatorname{im}(g-\mathbf{1}) \cap \operatorname{ker}(q)=0$, the unique minimal transformer involves $S=\operatorname{im}(g-\mathbf{1})$, and we set $s=\operatorname{dim}(S)$. If $S$ is not totally isotropic, the minimal number of reflections is $s$. If $S$ is totally isotropic, the minimal number of reflections is $>s$; if $v$ is any non-isotropic vector (therefore, outside $S$ ), the transformer for $\mathrm{R}(v) g$ (or $g \mathrm{R}(v)$ ) involves the subspace $S \oplus K v$ which is not totally isotropic; consequently, it is a product of $s+1$ reflections, and $g$ itself is a product of $s+2$ reflections. If $q$ is non-defective, $g$ cannot be a product of $s+1$ reflections, because the parity of the number of reflections is determined by $g$. On the contrary, if $q$ is defective, we have $\mathrm{R}(w)=\mathbf{1}_{V}$ for every non-isotropic $w \in \operatorname{ker}\left(\mathrm{b}_{q}\right)$, and the equality $g=\mathrm{R}(w) g$ proves that $g$ is a product of $s+1$ reflections.

In the difficult case $\operatorname{im}(g-\mathbf{1}) \cap \operatorname{ker}(q) \neq 0$, the dimension $s$ of a minimal transformer $(S, \phi)$ is $\operatorname{dim}(\operatorname{im}(g-\mathbf{1}))+\operatorname{dim}(\operatorname{im}(g-$ 1) $\cap \operatorname{ker}(q)$ ), and we can require $S$ not to be totally isotropic if and only if $\operatorname{ker}(g-\mathbf{1})^{\perp}$ is not totally isotropic; if it is not, the minimal number of reflections is $s$. On the contrary, if $\operatorname{ker}(g-\mathbf{1})^{\perp}$ is totally isotropic, the same is true for its subspace $\operatorname{ker}\left(\mathrm{b}_{q}\right)$; this means that $q$ is non-defective; and the same argument (involving $\mathrm{R}(v) g$ or $g \mathrm{R}(v)$ ) proves that the minimal number of reflections is $s+2$.

Remark. When the support $S$ of a transformer $(S, \phi)$ is totally isotropic, the dimension $s$ of $S$ is even, because $\phi$ is a nondegenerate and alternate bilinear form on $S$. There is a basis $\left(y_{1}, z_{1}, \ldots, y_{r}, z_{r}\right)$ of $S$ (where $r=s / 2$ ) such that $\phi\left(y_{i}, z_{i}\right)=1$ for $i=1,2, \ldots, r$, but $\phi\left(y_{i}, z_{j}\right)=0$ whenever $i \neq j$, and $\phi\left(y_{i}, y_{j}\right)=\phi\left(z_{i}, z_{j}\right)=0$ for all $i$ and $j$; and it is convenient to 
consider $\omega=\sum_{i=1}^{r} y_{i} \wedge z_{i}$ in $\bigwedge^{2}(S)$ because the transformation determined by $(S, \phi)$ is the transformation $\mathrm{F}(\omega)$ such that

$$
\forall x \in V, \quad \mathrm{~F}(\omega)(x)=x+\sum_{i=1}^{r}\left(\mathrm{~b}_{q}\left(x, y_{i}\right) z_{i}-\mathrm{b}_{q}\left(x, z_{i}\right) y_{i}\right) .
$$

If $q$ is non-degenerate, then $4 r=2 s \leq n$; therefore, a totally isotropic $S$ (such that $S \neq 0$ ) can appear only when $n \geq 4$. This explains that $s+2 \leq n$. Nevertheless, when $q$ is degenerate, it may happen that $s+2>n$, as in the following example.

Example. Let $(V, q)$ be the space with basis $\left(u_{1}, u_{2}, u_{3}\right)$ over $\mathbb{R}$, provided with the quadratic form $q$ such that $q\left(\xi_{1} u_{1}+\xi_{2} u_{2}+\right.$ $\left.\xi_{3} u_{3}\right)=\xi_{1} \xi_{2}$; thus $\operatorname{ker}(q)$ is the line $\mathbb{R} u_{3}$. Let $g$ be the orthogonal transformation such that

$$
g\left(\xi_{1} u_{1}+\xi_{2} u_{2}+\xi_{3} u_{3}\right)=\xi_{1}\left(u_{1}+u_{3}\right)+\xi_{2} u_{2}+\xi_{3} u_{3} .
$$

It is determined by the transformer $(S, \phi)$ such that $\left(u_{2}, u_{3}\right)$ is a basis of $S, \phi$ is alternate and $\phi\left(u_{2}, u_{3}\right)=1$; this agrees with (3.1). Therefore, when $g$ is expressed as a product of reflections, the minimal number of reflections is 4 . Let us calculate the transformer $(T, \psi)$ for $h=\mathrm{R}\left(u_{1}+u_{2}\right) g$. Since $T=\mathbb{R}\left(u_{1}+u_{2}\right) \oplus S$, we have $T=V ;$ since $\psi\left(u_{1}+u_{2}, u_{2}\right)=$ $\psi\left(u_{1}+u_{2}, u_{3}\right)=0$, we have $\psi\left(u_{1}, u_{2}\right)=0$ and $\psi\left(u_{1}, u_{3}\right)=-1$; the matrix $\Psi$ of $\psi$ in the basis $\left(u_{1}, u_{2}, u_{3}\right)$ is written below. In this example, it is easy to find a basis $\left(v_{1}, v_{2}, v_{3}\right)$ where the matrix $\Psi^{\prime}$ of $\psi$ is lower triangular; for instance,

$$
\left\{\begin{array}{l}
v_{1}=u_{1}+u_{2}+u_{3}, \\
v_{2}=u_{1}+2 u_{2}, \\
v_{3}=u_{1}+2 u_{2}-2 u_{3},
\end{array} \quad \Psi=\left(\begin{array}{ccc}
0 & 0 & -1 \\
1 & 0 & 1 \\
1 & -1 & 0
\end{array}\right), \quad \Psi^{\prime}=\left(\begin{array}{lll}
1 & 0 & 0 \\
3 & 2 & 0 \\
3 & 4 & 2
\end{array}\right) .\right.
$$

The result of this calculation is

$$
g=\mathrm{R}\left(u_{1}+u_{2}\right) \mathrm{R}\left(u_{1}+u_{2}+u_{3}\right) \mathrm{R}\left(u_{1}+2 u_{2}\right) \mathrm{R}\left(u_{1}+2 u_{2}-2 u_{3}\right) .
$$

There is an non-degenerate embedding $(W, \tilde{q})$ with a basis $\left(u_{1}, \ldots, u_{4}\right)$ such that $\tilde{q}\left(\sum_{i=1}^{4} \xi_{i} u_{i}\right)=\xi_{1} \xi_{2}+\xi_{3} \xi_{4}$. The extension $\tilde{g}$ maps $u_{4}$ to $u_{4}-u_{2}$; and (3.3) gives a decomposition of $\tilde{g}$ if the reflections operate on $W$.

Remark. When $K=\mathbb{Z} / 2 \mathbb{Z}$, the group $\mathrm{O}(V, q)$ is different from the subgroup $\mathrm{O}_{\mathrm{R}}(V, q)$ generated by the reflections in the following two exceptional cases (see (Helmstetter \& Micali, 2008), section 5.7). Dieudonné's exceptional case occurs when $V$ is the direct sum of $\operatorname{ker}(q)$ (perhaps reduced to 0) and a hyperbolic subspace of dimension 4 (with a basis $\left(u_{1}, \ldots, u_{4}\right)$ such that $\left.q\left(\sum_{i} \xi_{i} u_{i}\right)=\xi_{1} \xi_{2}+\xi_{3} \xi_{4}\right)$; in this case, the quotient $\mathrm{O}(V, q) / \mathrm{O}_{\mathrm{R}}(V, q)$ is a group of order 2 . The other case occurs when $V$ is the direct sum of $\operatorname{ker}(q)$ and a hyperbolic space of dimension 2 ; in this case, $\mathrm{O}(V, q) / \mathrm{O}_{\mathrm{R}}(V, q)$ is isomorphic to the additive group $\operatorname{ker}(q)$; it is exceptional only if $\operatorname{ker}(q) \neq 0$ (an eventuality which Dieudonné did not accept in (Dieudonné, 1958)). If we use (3.2) to define an orthogonal transformation $g$ over $\mathbb{Z} / 2 \mathbb{Z}$, then $g$ is not a product of reflections; and neither is its extension $\tilde{g}$ to a hyperbolic space of dimension 4.

\section{The Non-defective Case $\operatorname{dim}(\operatorname{ker}(q))=1$}

It is sensible to ask whether an orthogonal transformation $g$ of $(V, q)$ may admit several transformers. By means of a nondegenerate embedding $(W, \tilde{q})$, this question is easily reduced to the following one: does $\mathbf{1}_{V}$ admit several transformers, in other words, are there non-trivial transformers $(S, \phi)$ such that $S \subset \operatorname{ker}\left(\mathrm{b}_{q}\right)$ ? When $q$ is defective, the answer is obviously "yes" because the reflection associated with each non-isotropic $v \in \operatorname{ker}\left(\mathrm{b}_{q}\right)$ is equal to $\mathbf{1}_{V}$, and it admits the onedimensional transformer spanned by $v$. When $q$ is not defective, the condition $S \subset \operatorname{ker}\left(\mathrm{b}_{q}\right)$ implies that $\operatorname{dim}(S)$ is $\operatorname{even}$, and it can be satisfied by a non-trivial transformer if and only if $\operatorname{dim}\left(\operatorname{ker}\left(\mathrm{b}_{q}\right)\right) \geq 2$. Thus we have proved the following theorem.

Theorem 4.1. The correspondance between the orthogonal transformations and the transformers is bijective (only) in these two cases:

when $q$ is non-degenerate (in other words, $\operatorname{ker}\left(\mathrm{b}_{q}\right)=0$ );

when $q$ is non-defective and $\operatorname{dim}(\operatorname{ker}(q))=1$.

The non-defective case $\operatorname{dim}(\operatorname{ker}(q))=1$ deserves some attention because it can be used in the study of the affine isometries of an affine space $E$ provided with a non-degenerate quadratic form $\chi$. An affine space $E$ is a set on which a vector space $\vec{E}$ operates in a simply transitive way (by translations); the non-degenerate quadratic form $\chi$ is defined on $\vec{E}$; every affine transformation $g$ of $E$ has a linear part $\vec{g}$ in $\operatorname{GL}(\vec{E})$, and $g$ is an affine isometry if and only if $\vec{g} \in \mathrm{O}(\vec{E}, \chi)$; the set of all affine isometries is the group $\operatorname{Af}$.Iso $(E, \chi)$. For convenience, we set $n=\operatorname{dim}(E)+1$, and we suppose that $E=\vec{E}$; thus $\mathrm{O}(E, \chi)$ is the subgroup of all $g \in \operatorname{Af}$.Iso $E, \chi)$ such that $g(0)=0$. For every $a \in E$, let $a^{\sharp}$ be the linear form on $E$ such that $a^{\sharp}(b)=\mathrm{b}_{\chi}(a, b)$ for all $b \in E$; the mapping $a \longmapsto a^{\sharp}$ is a linear bijection $E \rightarrow E^{*}$, and the inverse bijection is denoted 
by $\ell \longmapsto \ell^{b}$; moreover, we define a dual quadratic form $\chi^{*}$ on $E^{*}$ by setting $\chi^{*}(\ell)=\chi\left(\ell^{b}\right)$. Let $V$ be the space of all affine forms $x: E \rightarrow K$; thus $E^{*}$ is the subspace of all $\ell \in V$ such that $\ell(0)=0$, and every $x \in V$ has a linear part $\vec{x} \in E^{*}$ such that $\vec{x}(a)=x(a)-x(0)$. Let $q$ be the quadratic form on $V$ defined by $q(x)=\chi^{*}(\vec{x})=\chi\left(\vec{x}^{b}\right)$. Thus $V$ is a space of dimension $n$ provided with a non-defective quadratic form $q$ such that $\operatorname{dim}(\operatorname{ker}(q))=1$; indeed, $\operatorname{ker}(q)$ is the set of all constant functions $E \rightarrow K$. Every affine transformation $g$ of $E$ determines a linear transformation $g^{\sharp}$ of $V$ which maps every $x \in V$ to the affine form $a \longmapsto x(g(a))$. From this definition, it follows that $\left(g_{1} g_{2}\right)^{\sharp}=g_{2}^{\sharp} g_{1}^{\sharp}$. Besides, $\operatorname{ker}\left(g^{\sharp}-\mathbf{1}\right) \supset \operatorname{ker}(q)$ because $g^{\sharp}$ leaves invariant every constant function $E \rightarrow K$. It is easy to prove that the mapping $g \longmapsto g^{\sharp}$ induces an anti-isomorphism from $\operatorname{Af}$.Iso $(E, \chi)$ onto $\mathrm{O}(V, q)$. The inverse anti-isomorphism is denoted by $h \longmapsto h^{b}$.

By this anti-isomorphism $b$, the reflections in $(V, q)$ are in bijection with the affine reflections in $(E, \chi)$; if $v$ is a nonisotropic element of $V$, the set of all $a \in E$ such that $v(a)=0$ is an affine hyperplane of $E$, and $(\mathrm{R}(v))^{b}$ is the affine reflection determined by this affine hyperplane:

$$
\forall a \in E, \quad(\mathrm{R}(v))^{b}(a)=a-\frac{v(a)}{q(v)} \vec{v}^{b} .
$$

Thus the decomposition into products of affine reflections in $\operatorname{Af}$.Iso $(E, \chi)$ is reduced to the decomposition into products of reflections in $\mathrm{O}(V, q)$.

Let $g$ be an element of Af.Iso $(E, \chi)$ (other than $\mathbf{1}_{E}$ ). We must find out whether $\operatorname{im}\left(g^{\sharp}-\mathbf{1}\right) \cap \operatorname{ker}(q)$ is reduced to 0 or not. If it is, there is a hyperplane $H$ of $V$ that contains $\operatorname{im}\left(g^{\sharp}-\mathbf{1}\right)$ but not $\operatorname{ker}(q)$; since $H$ does not contain $\operatorname{ker}(q)$, there is a point $p \in E$ such that $H$ is the subset of all $x \in V$ such that $x(p)=0$; and since $H$ contains $\operatorname{im}\left(g^{\sharp}-\mathbf{1}\right)$, we have $g^{\sharp}(H)=H$ and $g(p)=p$. Conversely, if $g(p)=p$ for some $p \in E$, then $g^{\sharp}(x)(p)=x(g(p))=x(p)$ for all $x \in V$, and $\left(g^{\sharp}-\mathbf{1}\right)(x)$ cannot be a constant function $\neq 0$. Therefore, the easy case $\operatorname{im}\left(g^{\sharp}-\mathbf{1}\right) \cap \operatorname{ker}(q)=0$ occurs if and only if $g(p)=p$ for some $p \in E$. If $g(p)=p$, then $g=T \vec{g} T^{-1}$ where $T$ is the translation $a \longmapsto a+p$, and the decomposition of $g$ into a product of affine reflections is reduced to the decomposition of $\vec{g}$ into a product of reflections in $\mathrm{O}(E, \chi)$.

Now we consider the difficult case $\operatorname{im}\left(g^{\sharp}-\mathbf{1}\right) \supset \operatorname{ker}(q)$. We have $g(a)=\vec{g}(a)+g(0)$ for all $a \in E$, and $g(0)$ is not in $\operatorname{im}\left(\vec{g}-\mathbf{1}_{E}\right)$ because the equality $g(0)=\vec{g}(b)-b$ is equivalent to $g(-b)=-b$, which is only possible in the above easy case. According to Theorem 2.2, we must find out whether $\operatorname{ker}\left(g^{\sharp}-\mathbf{1}\right)^{\perp}$ is totally isotropic or not; $\operatorname{since}$ it contains $\operatorname{ker}(q)$, it is determined by its image by the mapping $x \longmapsto \vec{x}^{\mathrm{b}}$. For all $x \in V$ and all $a \in E$, we have:

$$
\left(g^{\sharp}-\mathbf{1}\right)(x)(a)=\mathrm{b}_{\chi}\left(\vec{x}^{b},\left(\vec{g}-\mathbf{1}_{E}\right)(a)+g(0)\right) ;
$$

therefore, $x$ is in $\operatorname{ker}\left(g^{\sharp}-\mathbf{1}\right)$ if and only if $\vec{x}^{b}$ is orthogonal to $\operatorname{im}\left(\vec{g}-\mathbf{1}_{E}\right)$ and $g(0)$; and $y$ is in $\operatorname{ker}\left(g^{\sharp}-\mathbf{1}\right)^{\perp}$ if and only if $\vec{y}^{b}$ is in the direct sum of $\operatorname{im}\left(\vec{g}-\mathbf{1}_{E}\right)$ and the line $K g(0)$. Consequently, $\operatorname{ker}\left(g^{\sharp}-\mathbf{1}\right)^{\perp}$ is totally isotropic in $(V, q)$ if and only if $\operatorname{im}\left(\vec{g}-\mathbf{1}_{E}\right) \oplus K g(0)$ is totally isotropic in $(E, \chi)$.

We must also know how to deduce $s=\operatorname{dim}(S)$ from $d=\operatorname{dim}\left(\operatorname{im}\left(\vec{g}-\mathbf{1}_{E}\right)\right)$. The dimensions of $\operatorname{im}\left(\vec{g}-\mathbf{1}_{E}\right) \oplus K g(0)$ and $\operatorname{ker}\left(g^{\sharp}-\mathbf{1}\right)^{\perp}$ are $d+1$ and $d+2$. The dimension of $\operatorname{im}\left(g^{\sharp}-\mathbf{1}\right)$ is $d+1$ because of this fact: the sum of the dimensions of $\operatorname{ker}\left(g^{\sharp}-\mathbf{1}\right)$ and $\operatorname{im}\left(g^{\sharp}-\mathbf{1}\right)$ is $n$, but the sum of the dimensions of $\operatorname{ker}\left(g^{\sharp}-\mathbf{1}\right)$ and $\operatorname{ker}\left(g^{\sharp}-\mathbf{1}\right)^{\perp}$ is $n+1$ because $\operatorname{ker}\left(g^{\sharp}-\mathbf{1}\right) \supset \operatorname{ker}(q)$. From (2.7) we deduce $s=d+2$. Since $s \leq n$, we have $d \leq n-2$, in agreement with $g(0) \notin \operatorname{im}\left(\vec{g}-\mathbf{1}_{E}\right)$.

When $\operatorname{im}\left(\vec{g}-\mathbf{1}_{E}\right) \oplus K g(0)$ is totally isotropic, may it occur that $s+2>n$ ? The example below shows that it occurs when $n=3$ and $d=0$. But other occurences are only possible with $d>0$. Since $\chi$ is non-degenerate, we have $2(d+1) \leq n-1$ when $\operatorname{im}\left(\vec{g}-\mathbf{1}_{E}\right) \oplus K g(0)$ is totally isotropic; moreover, $d$ is even like $s$; consequently, $n \geq 7$ if $d>0$; and it is easy to realize that $s+2<n$ when $n \geq 7$ and $2(d+1) \leq n-1$.

Example. Let $(E, \chi)$ be the vector space with basis $\left(e_{1}, e_{2}\right)$ over $\mathbb{R}$, where $\chi\left(\xi_{1} e_{1}+\xi_{2} e_{2}\right)=\xi_{1} \xi_{2}$; and let $g$ be the translation of vector $e_{1}$. In general, a translation is a product of two reflections; but here we shall need four reflections because $e_{1}$ is isotropic. With the notation used just above, we have $n=3, d=0$ because $\vec{g}=\mathbf{1}_{E}$, and $s=2$; but since $S$ will prove to be totally isotropic in $(V, q)$, we need $s+2$ reflections. Let $u_{1}, u_{2}$ and $u_{3}$ be the affine forms that map every $\xi_{1} e_{1}+\xi_{2} e_{2}$ respectively to $\xi_{1}, \xi_{2}$ and 1 ; thus $\left(u_{1}, u_{2}, u_{3}\right)$ is a basis of $V$. The mapping $x \longmapsto \vec{x}^{b}$ maps $u_{1}, u_{2}, u_{3}$ respectively to $e_{2}, e_{1}, 0$; consequently, $q\left(\xi_{1} u_{1}+\xi_{2} u_{2}+\xi_{3} u_{3}\right)=\xi_{1} \xi_{2}$. An easy calculation shows that $g^{\sharp}$ maps $u_{1}, u_{2}, u_{3}$ respectively to $u_{1}+u_{3}, u_{2}$, $u_{3}$; thus $g^{\sharp}$ coincides with the orthogonal transformation defined by (3.2). We already know that $S$ is spanned by $\left(u_{2}, u_{3}\right)$, and we translate (3.3) here in this way:

$$
g=\left(\mathrm{R}\left(u_{1}+2 u_{2}-2 u_{3}\right)\right)^{\mathrm{b}}\left(\mathrm{R}\left(u_{1}+2 u_{2}\right)\right)^{b}\left(\mathrm{R}\left(u_{1}+u_{2}+u_{3}\right)\right)^{\mathrm{b}}\left(\mathrm{R}\left(u_{1}+u_{2}\right)\right)^{\mathrm{b}}
$$

$\left(\mathrm{R}\left(u_{1}+2 u_{2}-2 u_{3}\right)\right)^{\mathrm{b}}\left(\mathrm{R}\left(u_{1}+2 u_{2}\right)\right)^{\mathrm{b}}$ is the translation of vector $2 e_{1}+e_{2}$, and $\left(\mathrm{R}\left(u_{1}+u_{2}+u_{3}\right)\right)^{\mathrm{b}}\left(\mathrm{R}\left(u_{1}+u_{2}\right)\right)^{\mathrm{b}}$ is the translation of vector $-e_{1}-e_{2}$. 


\section{An Algorithm of Triangularization}

Theorem 3.3 states that there are bases $\left(v_{1}, \ldots, v_{s}\right)$ of $S$ where the matrix of $\phi$ is lower triangular, provided that $\phi$ is not alternate; this must be proved when $s \geq 2$, and to prove it, I propose an algorithm of triangularization. There are two standard versions of this algorithm; the left side version calculates the vectors $v_{i}$ in the increasing order of the indices $i$; as a by-product, it gives a basis of $\operatorname{RKer}(\phi)$. When the dimension $t$ of $\operatorname{LKer}(\phi)$ and $\operatorname{RKer}(\phi)$ is $\neq 0$, it gives a triangularizing basis $\left(v_{1}, \ldots, v_{s}\right)$ where $\phi\left(v_{i}, v_{i}\right) \neq 0$ for $i=1,2, \ldots, s-t$, and $\left(v_{s-t+1}, \ldots, v_{s}\right)$ is a basis of $\operatorname{RKer}(\phi)$. The right side version calculates the vectors $v_{i}$ in the decreasing order of the indices, and when $t \neq 0$, then $\left(v_{1}, \ldots, v_{t}\right)$ is a basis of $\operatorname{LKer}(\phi)$. Each version requires $s-1$ steps if $t=0$, and $s-t$ steps if $t \geq 1$.

The space $(S, \phi)$ is given by a basis $\left(u_{1}, \ldots, u_{s}\right)$ and the matrix of $\phi$ in this basis. When the $k$-th step of the left side algorithm begins, we know a sequence $\left(v_{1}, \ldots, v_{k-1}, \dot{v}_{k}\right)$ such that $\phi\left(v_{i}, v_{i}\right) \neq 0$ for $i=1,2, \ldots, k-1, \phi\left(\dot{v}_{k}, \dot{v}_{k}\right) \neq 0$, $\phi\left(v_{i}, v_{j}\right)=0$ whenever $i<j$, and $\phi\left(v_{i}, \dot{v}_{k}\right)=0$ for $i=1,2, \ldots, k-1$. In particular, the first step begins with a vector $\dot{v}_{1}$ such that $\phi\left(\dot{v}_{1}, \dot{v}_{1}\right) \neq 0$; such a vector $\dot{v}_{1}$ exists because $\phi$ is not alternate. In general, the instructions of this algorithm order to set $v_{k}=\dot{v}_{k}$; but sometimes, the vector $\dot{v}_{k}$ must be "corrected" (replaced by a suitable $v_{k}$ ); the "correction procedure" (the instruction ((8)) below) is the only phase that may fail when $K \cong \mathbb{Z} / 2 \mathbb{Z}$. The $k$-th step is performed according to the following eight instructions.

((1)) In the basis $\left(u_{1}, u_{2}, \ldots, u_{s}\right)$ we choose a subsequence $\left(x_{1}, x_{2}, \ldots, x_{s-k}\right)$ such that $\left(v_{1}, \ldots, v_{k-1}, \dot{v}_{k}, x_{1}, \ldots, x_{s-k}\right)$ is a basis of $S$.

((2)) For $j=1,2, \ldots, s-k$, and as long as the "stop rule" (written just below) does not interrupt the calculations, we calculate the scalars $\xi_{1}, \ldots, \xi_{k}$ that let the vector $y_{j}=\xi_{1} v_{1}+\cdots+\xi_{k-1} v_{k-1}+\xi_{k} \dot{v}_{k}+x_{j}$ satisfy the following conditions:

$$
\phi\left(v_{1}, y_{j}\right)=\phi\left(v_{2}, y_{j}\right)=\cdots=\phi\left(v_{k-1}, y_{j}\right)=\phi\left(\dot{v}_{k}, y_{j}\right)=0 ;
$$

the properties of the sequence $\left(v_{1}, \ldots, \dot{v}_{k}\right)$ show that (5.1) is a regular system of $k$ linear equations with a lower triangular matrix; therefore, the calculation of $\xi_{1}, \ldots, \xi_{k}$ is easy. When $k=s-1$, we have to calculate only one vector $y_{1}$, and then we go to ((3)). When $k \leq s-2$, the stop rule interrupts the calculations in these two cases:

when we find a vector $y_{j}$ such that $\phi\left(y_{j}, y_{j}\right) \neq 0$, we go to ((4));

when we find two vectors $y_{i}$ and $y_{j}$ such that $\phi\left(y_{i}, y_{i}\right)=\phi\left(y_{j}, y_{j}\right)=0$ and $\phi\left(y_{i}, y_{j}\right)+\phi\left(y_{j}, y_{i}\right) \neq 0$, we go to ((5)).

When the stop rule never interrupts the calculations, we go to ((6)).

((3)) When $k=s-1$, we set $v_{s-1}=\dot{v}_{s-1}$ and $v_{s}=y_{1}$. Thus we have found a triangularizing basis $\left(v_{1}, \ldots, v_{s}\right)$. If $\phi\left(v_{s}, v_{s}\right) \neq 0$, then $\phi$ is non-degenerate. If $\phi\left(v_{s}, v_{s}\right)=0$, then $\operatorname{RKer}(\phi)$ is the line spanned by $v_{s}$.

In the next instructions, we have $k \leq s-2$.

((4)) When $\phi\left(y_{j}, y_{j}\right) \neq 0$, we set $v_{k}=\dot{v}_{k}$ and $\dot{v}_{k+1}=y_{j}$, and we start the $(k+1)$-th step (we return to ((1)) where we replace $k$ with $k+1)$.

((5)) When $\phi\left(y_{i}, y_{i}\right)=\phi\left(y_{j}, y_{j}\right)=0$ and $\phi\left(y_{i}, y_{j}\right)+\phi\left(y_{j}, y_{i}\right) \neq 0$, we set $v_{k}=\dot{v}_{k}$ and $\dot{v}_{k+1}=y_{i}+y_{j}$, and we start the $(k+1)$-th step.

((6)) When the stop rule never interrupts the calculations, the restriction of $\phi$ to the subspace spanned by $\left(y_{1}, \ldots, y_{s-k}\right)$ (that is $\left.\mathrm{R}_{\phi}^{\perp}\left(v_{1}, \ldots, \dot{v}_{k}\right)\right)$ is alternate. If there is a couple $(i, j)$ such that $\phi\left(y_{i}, y_{j}\right) \neq 0$, we go to ((8))). If all $\phi\left(y_{i}, y_{j}\right)$ (with $i, j \in\{1,2, \ldots, s-k\})$ vanish, we go to ((7)).

((7)) If all $\phi\left(y_{i}, y_{j}\right)$ vanish, then we set $v_{k}=\dot{v}_{k}, v_{k+1}=y_{1}, v_{k+2}=y_{2}, \ldots, v_{s}=y_{s-k}$. Thus we have found a triangularizing basis $\left(v_{1}, \ldots, v_{s}\right)$, where $\left(v_{k+1}, \ldots, v_{s}\right)$ is a basis of $\operatorname{RKer}(\phi)$; therefore, $t=s-k$.

((8)) Let $(i, j)$ be a couple (with $i \neq j$ ) such that

$$
\phi\left(y_{i}, y_{i}\right)=\phi\left(y_{j}, y_{j}\right)=0 \quad \text { and } \phi\left(y_{i}, y_{j}\right)=-\phi\left(y_{j}, y_{i}\right) \neq 0 .
$$

We look for scalars $\kappa, \lambda, \mu$ that ensure the three properties required from the vectors $v_{k}=\dot{v}_{k}+\kappa y_{i}$ and $\dot{v}_{k+1}=\dot{v}_{k}+\lambda y_{i}+\mu y_{j}$. Here are these properties:

$$
\begin{aligned}
\phi\left(v_{k}, \dot{v}_{k+1}\right) & =\phi\left(\dot{v}_{k}, \dot{v}_{k}\right)+\kappa \phi\left(y_{i}, \dot{v}_{k}\right)+\kappa \mu \phi\left(y_{i}, y_{j}\right)=0, \\
\phi\left(v_{k}, v_{k}\right) & =\phi\left(\dot{v}_{k}, \dot{v}_{k}\right)+\kappa \phi\left(y_{i}, \dot{v}_{k}\right) \neq 0, \\
\phi\left(\dot{v}_{k+1}, \dot{v}_{k+1}\right) & =\phi\left(\dot{v}_{k}, \dot{v}_{k}\right)+\lambda \phi\left(y_{i}, \dot{v}_{k}\right)+\mu \phi\left(y_{j}, \dot{v}_{k}\right) \neq 0 .
\end{aligned}
$$

(8a) If $\phi\left(y_{i}, \dot{v}_{k}\right)=0$, the condition (5.4) is void. We set $\lambda=0$, we choose an invertible $\mu$ compatible with (5.5), and we calculate $\kappa$ by means of (5.3). When $v_{k}$ and $\dot{v}_{k+1}$ have been calculated, we start the $(k+1)$-th step. 
(8b) If $\phi\left(y_{i}, \dot{v}_{k}\right) \neq 0$, we choose an invertible $\kappa$ compatible with (5.4), we calculate $\mu$ by means of (5.3), and we choose $\lambda$ compatible with (5.5); in general, the choice $\lambda=0$ is correct. When $v_{k}$ and $\dot{v}_{k+1}$ have been calculated, we start the $(k+1)$-th step. If $\phi\left(y_{i}, \dot{v}_{k}\right) \neq 0$ and $\phi\left(y_{j}, \dot{v}_{k}\right)=0$, it is preferable (but not indispensable) to permute $i$ and $j$ and to apply (8a) instead of (8b).

These instructions involve the correction procedure $((8))$ as rarely as possible (it is involved only when the restriction of $\phi$ to $\mathrm{R}_{\phi}^{\perp}\left(v_{1}, \ldots, \dot{v}_{k}\right)$ is alternate and $\left.\neq 0\right)$; this choice is suggested by an algorithm elaborated for a similar problem which involves a very painful correction procedure. Since here the correction procedure is not so painful, it is acceptable to modify the stop rule in such a way that ((8)) is involved as frequently as possible. When $k \leq s-2$, the new stop rule interrupts the calculations in ((2)) as soon as we meet a non-zero $\phi\left(y_{i}, y_{j}\right)$; when $i=j$, we go to ((4)); when $i \neq j$ and $\phi\left(y_{i}, y_{i}\right)=\phi\left(y_{j}, y_{j}\right)=0$, we go to ((5)), except when (5.2) is true; when (5.2) is true, we go to ((8)). Thus the instruction ((6)) becomes superfluous; if the new stop rule never interrupts the calculation, the restriction of $\phi$ to $\mathrm{R}_{\phi}^{\perp}\left(v_{1}, \ldots, \dot{v}_{k}\right)$ is completely null, and we go directly to ((7))).

The right side algorithm requires symmetric instructions. The $k$-th step starts with a sequence $\left(\dot{v}_{s-k+1}, v_{s-k+2}, \ldots, v_{s}\right)$ satisfying obvious conditions. In the instruction ((2)), we set $y_{j}=x_{j}+\xi_{1} \dot{v}_{s-k+1}+\xi_{2} v_{s-k+2}+\cdots+\xi_{k} v_{s}$, and the unknown scalars $\xi_{1}, \ldots, \xi_{k}$ are determined by a system of $k$ liner equations with an upper triangular matrix. In the correction procedure ((8)), we set $v_{s-k+1}=\kappa y_{i}+\dot{v}_{s-k+1}$ and $\dot{v}_{s-k}=\lambda y_{i}+\mu y_{j}+\dot{v}_{s-k+1}$; and the unknown scalars $\kappa, \lambda, \mu$ must satisfy

$$
\begin{aligned}
\phi\left(\dot{v}_{s-k}, v_{s-k+1}\right) & =\kappa \phi\left(\dot{v}_{s-k+1}, y_{i}\right)-\kappa \mu \phi\left(y_{i}, y_{j}\right)+\phi\left(\dot{v}_{s-k+1}, \dot{v}_{s-k+1}\right)=0, \\
\phi\left(v_{s-k+1}, v_{s-k+1}\right) & =\kappa \phi\left(\dot{v}_{s-k+1}, y_{i}\right)+\phi\left(\dot{v}_{s-k+1}, \dot{v}_{s-k+1}\right) \neq 0, \\
\phi\left(\dot{v}_{s-k}, \dot{v}_{s-k}\right) & =\lambda \phi\left(\dot{v}_{s-k+1}, y_{i}\right)+\mu \phi\left(\dot{v}_{s-k+1}, y_{j}\right)+\phi\left(\dot{v}_{s-k+1}, \dot{v}_{s-k+1}\right) \neq 0 .
\end{aligned}
$$

The left and right side versions are the ordered versions. But there are plenty of disordered versions where the vectors of a triangularizing basis are calculated in an arbitrary disorder; there is only one restriction in the choice of this disorder when $t \geq 2$ : the last step produces simultaneously $t$ isotropic vectors which give a connected subsequence in the resulting basis $\left(v_{1}, \ldots v_{s}\right)$ (not necessarily at the beginning or at the end). Lemma 1.3 (which involves two subspaces $U_{1}$ and $U_{2}$ of $S$ on which $\phi$ is non-degenerate) is the foundation of all these versions; the left side version uses it when $U_{2}=0$, the right side version when $U_{1}=0$, and the disordered versions use it in its full generality. There is an example of disordered algorithm in Section 7.

\section{Orthogonal Transformations Inside $(S, \phi)$}

The notation is the same as in Section 5; here we emphasize the quadratic form $q$ on $S$ such that $q(y)=\phi(y, y)$ for all $y \in S$. When $T$ is a subspace of $S$, the notation $(T, \phi)$ means the subspace $T$ provided with the restriction of $\phi$ to $T$. When this restriction is non-degenerate, $(T, \phi)$ is a transformer for $(S, q)$, and induces an orthogonal transformation $g$ on $S$ such that $\operatorname{im}\left(g-\mathbf{1}_{S}\right) \subset T$. Besides, Lemma 1.3 implies $S=T \oplus \mathrm{R}_{\phi}^{\perp}(T)=\mathrm{L}_{\phi}^{\perp}(T) \oplus T$.

Theorem 6.1. If the restriction of $\phi$ to $T$ is non-degenerate, the orthogonal transformation $g$ induced by $(T, \phi)$ maps $\mathrm{R}_{\phi}^{\perp}(T)$ onto $\mathrm{L}_{\phi}^{\perp}(T)$; moreover,

$$
\forall x, y \in \mathrm{R}_{\phi}^{\perp}(T), \quad \phi(g(x), g(y))=\phi(x, y) .
$$

Proof. When $\mathrm{b}_{q}(x, y)=\phi(x, y)+\phi(y, x)$, the equation (2.1) gives

$$
\forall x \in S, \forall y \in T, \quad \phi(g(x), y)=-\phi(y, x) ;
$$

therefore, $g(x)$ is in $\mathrm{L}_{\phi}^{\perp}(T)$ if and only if $x$ is in $\mathrm{R}_{\phi}^{\perp}(T)$. For all $x, y \in S$,

$$
\phi(x, g(y))-\phi\left(g^{-1}(x), y\right)=\phi(x, g(y)-y)-\phi\left(g^{-1}(x)-x, y\right) ;
$$

both $g(y)-y$ and $g^{-1}(x)-x$ belong to $T$; when $x$ and $y$ belong respectively to $\mathrm{L}_{\phi}^{\perp}(T)$ and $\mathrm{R}_{\phi}^{\perp}(T)$, then $\phi(x, g(y)-y)$ and $\phi\left(g^{-1}(x)-x, y\right)$ vanish, and $\phi(x, g(y))=\phi\left(g^{-1}(x), y\right)$ in accordance with (6.1).

The equality (6.1) is also true when $x$ and $y$ belong to $T$ : see Theorem 2.1, formula (2.5); in general, it is false when $x$ and $y$ are arbitrary elements of $S$.

When $\phi$ is degenerate, Theorem 6.1 gives a property of $\operatorname{LKer}(\phi)$ and $\operatorname{RKer}(\phi)$; as in Section 5, their dimension is denoted by $t$. The restriction of $\phi$ to a subspace $T$ of dimension $s-t$ is non-degenerate if and only if $\operatorname{LKer}(\phi) \cap T=T \cap \operatorname{RKer}(\phi)=0$; when it is non-degenerate, then $\operatorname{LKer}(\phi)=\mathrm{L}_{\phi}^{\perp}(T)$ and $\operatorname{RKer}(\phi)=\mathrm{R}_{\phi}^{\perp}(T)$; therefore, the orthogonal transformation induced by $(T, \phi)$ maps $\operatorname{RKer}(\phi)$ bijectively onto $\operatorname{LKer}(\phi)$.

Theorem 6.1 also enables us to perform operations on a triangularizing basis $\left(v_{1}, \ldots, v_{s}\right)$ of $(S, \phi)$. Let us consider a subsequence $\left(v_{h+1}, v_{h+2}, \ldots, v_{h+c+d}\right)$ where $h, c, d$ are integers such that $c>0, d>0$ and $0 \leq h \leq s-c-d$. Let $T_{1}$ be 
the subspace spanned by $\left(v_{h+1}, \ldots, v_{h+c}\right), T_{2}$ the subspace spanned by $\left(v_{h+c+1}, \ldots, v_{h+c+d}\right)$, and $S^{\prime}=T_{1} \oplus T_{2}$. When $v_{j}$ is never isotropic for $h<j \leq h+c$, let $g_{1}$ be the orthogonal transformation of $\left(S^{\prime}, q\right)$ induced by the transformer $\left(T_{1}, \phi\right)$; it is equal to the product of the reflections $\mathrm{R}\left(v_{j}\right)$ with $j=h+1, h+2, \ldots, h+c$. And when $v_{j}$ is never isotropic for $h+c<j \leq h+c+d$, let $g_{2}$ be the orthogonal transformation of $\left(S^{\prime}, q\right)$ induced by the reverse transformer $\left(T_{2}, \phi^{\dagger}\right)$; it is the product of the reflections $\mathrm{R}\left(v_{j}\right)$ with $j=h+c+d, h+c+d-1, \ldots, h+c+1$. We obtain another triangularizing basis if we replace the subsequence $\left(v_{h+1}, \ldots, v_{h+c+d}\right)$ with

$$
\left(g_{1}\left(v_{h+c+1}\right), \ldots, g_{1}\left(v_{h+c+d}\right), v_{h+1}, \ldots, v_{h+c}\right) \quad \text { or } \quad\left(v_{h+c+1}, \ldots, v_{h+c+d}, g_{2}\left(v_{h+1}\right), \ldots, g_{2}\left(v_{h+c}\right)\right) .
$$

\section{Examples}

\section{First example: a rotation in a euclidean plane}

Let $(V, q)$ be a euclidean plane over $\mathbb{R}$, provided with a basis $\left(e_{1}, e_{2}\right)$ such that $q\left(\xi_{1} e_{1}+\xi_{2} e_{2}\right)=\xi_{1}^{2}+\xi_{2}^{2}$, whence $\mathrm{b}_{q}\left(\xi_{1} e_{1}+\right.$ $\left.\xi_{2} e_{2}, \zeta_{1} e_{1}+\zeta_{2} e_{2}\right)=2\left(\xi_{1} \zeta_{1}+\xi_{2} \zeta_{2}\right)$. Let $g$ be the rotation of angle $2 \theta$ such that $\sin (\theta) \neq 0$ (so that $g \neq \mathbf{1}$ ); its matrix $G$ is written below. Since $g-\mathbf{1}$ is a bijection $V \rightarrow V$, the formula $(2.1)$ gives $\phi(x, y)=-\mathrm{b}_{q}\left((g-\mathbf{1})^{-1}(x), y\right)$; therefore, the matix $\Phi$ of $\phi$ is obtained by transposition of $-2(G-\mathbf{1})^{-1}$ :

$$
G=\left(\begin{array}{cc}
\cos (2 \theta) & -\sin (2 \theta) \\
\sin (2 \theta) & \cos (2 \theta)
\end{array}\right), \quad \Phi=\frac{1}{\sin (\theta)}\left(\begin{array}{cc}
\sin (\theta) & \cos (\theta) \\
-\cos (\theta) & \sin (\theta)
\end{array}\right) .
$$

Let us consider $v_{1}=\cos (\lambda) e_{1}+\sin (\lambda) e_{2}$ and $v_{2}=\cos (\mu) e_{1}+\sin (\mu) e_{2}$; which are the couples $(\lambda, \mu)$ for which $g=$ $\mathrm{R}\left(v_{1}\right) \mathrm{R}\left(v_{2}\right)$ ? According to Corollary 3.2, this is true if and only if $\phi\left(v_{1}, v_{2}\right)=0$; let us verify that this equation agrees with the answer that has been known for already more than 2000 years:

$$
\phi\left(v_{1}, v_{2}\right)=(\cos (\lambda) \sin (\lambda)) \Phi\left(\begin{array}{c}
\cos (\mu) \\
\sin (\mu)
\end{array}\right)=\frac{\sin (\theta-\lambda+\mu)}{\sin (\theta)} ;
$$

thus $g=\mathrm{R}\left(v_{1}\right) \mathrm{R}\left(v_{2}\right)$ if and only if $\lambda-\mu=\theta$ modulo $\pi$.

\section{Second example with a correction procedure}

Here $(V, q)$ is given by the basis $\left(e_{1}, e_{2}, e_{3}, e_{4}\right)$ over $\mathbb{R}$, and the quadratic form $q$ such that $q\left(\sum_{i=1}^{4} \xi_{i} e_{i}\right)=\xi_{1} \xi_{2}+\xi_{3} \xi_{4}$. Let us apply the left and right side algorithms to the orthogonal transformation $g$ of $(V, q)$ described by the matrix $G$ just below. This matrix $G$ determines over the field $\mathbb{Z} / 2 \mathbb{Z}$ an orthogonal transformation that is not a product of reflections (it belongs to Dieudonné's exceptional case). The image of $g-\mathbf{1}$ is the subspace $S$ spanned by $\left(e_{1}, e_{3}, e_{4}\right) ; g-\mathbf{1}$ maps $e_{3}-e_{4}, e_{2}-e_{3}$, $-e_{2}$ respectively to $e_{1}, e_{3}, e_{4}$, and the matrix $\Phi$ of $\phi$ in the basis $\left(e_{1}, e_{3}, e_{4}\right)$ easily follows:

$$
G=\left(\begin{array}{cccc}
1 & 0 & 0 & -1 \\
0 & 1 & 0 & 0 \\
0 & 0 & 0 & -1 \\
0 & -1 & -1 & 0
\end{array}\right), \quad \Phi=\left(\begin{array}{ccc}
0 & 1 & -1 \\
-1 & 0 & 1 \\
1 & 0 & 0
\end{array}\right)
$$

Let us begin the left side algorithm with $\dot{v}_{1}=e_{3}+e_{4}$. Since this choice of $\dot{v}_{1}$ is also acceptable for the field $\mathbb{Z} / 2 \mathbb{Z}$, we are sure to need a correction; indeed, the predictable failure of the algorithm over $\mathbb{Z} / 2 \mathbb{Z}$ can be explained only by its failure during a correction procedure. By means of the basis $\left(\dot{v}_{1}, e_{1}, e_{3}\right)$ of $S$, we start the calculation of a basis $\left(y_{1}, y_{2}\right)$ of $\mathrm{R}_{\phi}^{\perp}\left(\dot{v}_{1}\right)$. For $y_{1}=\xi_{1} \dot{v}_{1}+e_{1}$, the condition $\phi\left(\dot{v}_{1}, y_{1}\right)=0$ gives $\xi_{1}=0$, whence $y_{1}=e_{1}$ and $\phi\left(y_{1}, y_{1}\right)=0$. Therefore, we also calculate $y_{2}=\xi_{1} \dot{v}_{1}+e_{3}$; the condition $\phi\left(\dot{v}_{1}, y_{2}\right)=0$ gives again $\xi_{1}=0$, whence $y_{2}=e_{3}, \phi\left(y_{2}, y_{2}\right)=0$, and $\phi\left(y_{1}, y_{2}\right)=-\phi\left(y_{2}, y_{1}\right)=1$. Since this agrees with (5.2), a correction is necessary; since $\phi\left(y_{1}, \dot{v}_{1}\right)=0$ and $\phi\left(y_{2}, \dot{v}_{1}\right)=1$, we follow (8a) in the instruction ((8)). We set $v_{1}=\dot{v}_{1}+\kappa y_{1}$ (whence $\left.\phi\left(v_{1}, v_{1}\right)=1\right)$ and $\dot{v}_{2}=\dot{v}_{1}+\mu y_{2}$; the condition $\phi\left(v_{1}, \dot{v}_{2}\right)=0$ gives $1+\kappa \mu=0$, and the condition $\phi\left(\dot{v}_{2}, \dot{v}_{2}\right) \neq 0$ gives $1+\mu \neq 0$. As it was predictable, these two conditions cannot be satisfied over the field $\mathbb{Z} / 2 \mathbb{Z}$. But over $\mathbb{R}$, they are satisfied with $\mu=1$ and $\kappa=-1$. Consequently, we start the second step of the algorithm with $v_{1}=-e_{1}+e_{3}+e_{4}$ and $\dot{v}_{2}=2 e_{3}+e_{4}$.

Since $\left(v_{1}, \dot{v}_{2}, e_{4}\right)$ is a basis of $S$, we set $y_{1}=\xi_{1} v_{1}+\xi_{2} \dot{v}_{2}+e_{4}$ and we calculate $\xi_{1}$ and $\xi_{2}$ with the equations $\phi\left(v_{1}, y_{1}\right)=$ $\phi\left(\dot{v}_{2}, y_{1}\right)=0$, which give $\xi_{1}+2=3 \xi_{1}+2 \xi_{2}+2=0$, whence $\xi_{1}=-2$ and $\xi_{2}=2$. According to the instruction ((3)), we set $v_{2}=\dot{v}_{2}$ and $v_{3}=y_{1}=-2 v_{1}+2 v_{2}+e_{4}$. Here is the basis $\left(v_{1}, v_{2}, v_{3}\right)$ and the matrix $\Phi^{\prime}$ of $\phi$ in this basis:

$$
\left\{\begin{array}{l}
v_{1}=-e_{1}+e_{3}+e_{4}, \\
v_{2}=2 e_{3}+e_{4}, \\
v_{3}=2 e_{1}+2 e_{3}+e_{4},
\end{array} \quad \Phi^{\prime}=\left(\begin{array}{lll}
1 & 0 & 0 \\
3 & 2 & 0 \\
3 & 4 & 2
\end{array}\right) .\right.
$$


The conclusion of this calculation is $g=\mathrm{R}\left(v_{1}\right) \mathrm{R}\left(v_{2}\right) \mathrm{R}\left(v_{3}\right)$.

Now let us start the right side algorithm with $\dot{v}_{3}=e_{3}+e_{4}$ and the basis $\left(e_{1}, e_{3}, \dot{v}_{3}\right)$ of $S$. The calculation of $y_{1}=e_{1}+\xi_{1} \dot{v}_{3}$ such that $\phi\left(y_{1}, \dot{v}_{3}\right)=0$ gives $\xi_{1}=0$ and $y_{1}=e_{1}$. Therefore, we also calculate $y_{2}=e_{3}+\xi_{1} \dot{v}_{3}$ such that $\phi\left(y_{2}, \dot{v}_{3}\right)=0$; we find $\xi_{1}=-1$ and $y_{2}=-e_{4}$. Thus $\phi\left(y_{1}, y_{1}\right)=\phi\left(y_{2}, y_{2}\right)=0$ and $\phi\left(y_{1}, y_{2}\right)=-\phi\left(y_{2}, y_{1}\right)=1$; and a correction is necessary. Since $\phi\left(\dot{v}_{3}, y_{1}\right)=0$ and $\phi\left(\dot{v}_{3}, y_{2}\right)=-1$, we set $v_{3}=\kappa y_{1}+\dot{v}_{3}\left(\right.$ whence $\left.\phi\left(v_{3}, v_{3}\right)=1\right)$ and $\dot{v}_{2}=\mu y_{2}+\dot{v}_{3}$. The conditions $\phi\left(\dot{v}_{2}, v_{3}\right)=0$ and $\phi\left(\dot{v}_{2}, \dot{v}_{2}\right) \neq 0$ give $-\kappa \mu+1=0$ and $-\mu+1 \neq 0$; they are satisfied with $\mu=\kappa=-1$. Thus we start the second step with $\dot{v}_{2}=e_{3}+2 e_{4}$ and $v_{3}=-e_{1}+e_{3}+e_{4}$, and with the basis $\left(e_{4}, \dot{v}_{2}, v_{3}\right)$ of $S$. We must calculate $y_{1}=e_{4}+\xi_{1} \dot{v}_{2}+\xi_{2} v_{3}$ with the conditions $\phi\left(y_{1}, \dot{v}_{2}\right)=\phi\left(y_{1}, v_{3}\right)=0$; they give the equations $2 \xi_{1}+3 \xi_{2}=-1+\xi_{2}=0$, and determine $\xi_{2}=1$ and $\xi_{1}=-3 / 2$. Here is the final result of this calculation:

$$
\left\{\begin{array}{l}
v_{1}=-e_{1}-\frac{1}{2} e_{3}-e_{4}, \\
v_{2}=e_{3}+2 e_{4}, \\
v_{3}=-e_{1}+e_{3}+e_{4},
\end{array} \quad \Phi^{\prime}=\left(\begin{array}{ccc}
1 / 2 & 0 & 0 \\
-2 & 2 & 0 \\
-3 / 2 & 3 & 1
\end{array}\right) .\right.
$$

As above, $g=\mathrm{R}\left(v_{1}\right) \mathrm{R}\left(v_{2}\right) \mathrm{R}\left(v_{3}\right)$.

\section{Third example (an ordinary example)}

Let $(V, q)$ be the space over $\mathbb{R}$ determined by the orthogonal basis $\left(e_{1}, \ldots, e_{6}\right)$ such that $q\left(e_{i}\right)=1$ for $i=1,2,3,4$, and $q\left(e_{i}\right)=-1$ for $i=5,6$; and let $g$ be the orthogonal transofrmation of $(V, q)$ given by the following matrix:

$$
G=\left(\begin{array}{cccccc}
3 / 10 & -3 / 5 & -4 / 5 & 2 / 5 & 0 & -1 / 2 \\
-2 / 5 & -1 / 5 & 2 / 5 & 4 / 5 & 0 & 0 \\
-1 & 0 & -1 & 0 & 0 & -1 \\
-1 & -2 & 0 & -1 & 2 & -1 \\
1 / 5 & -2 / 5 & 4 / 5 & -2 / 5 & 1 & 1 \\
-11 / 10 & -9 / 5 & -2 / 5 & -4 / 5 & 2 & -3 / 2
\end{array}\right) .
$$

The kernel of $g-\mathbf{1}$ is spanned by $2 e_{1}-e_{2}-e_{3}$ and $e_{1}-e_{2}-e_{4}+2 e_{5}-e_{6}$. There are well known algorithms to find a convenient basis $\left(u_{1}, \ldots, u_{4}\right)$ of $S=\operatorname{im}(g-\mathbf{1})$; then the matrix $\Phi$ of $\phi$ in this basis is calculated with (2.1):

$$
\left\{\begin{array}{ll}
u_{1}=(g-\mathbf{1})\left(-2 e_{1}-e_{4}-2 e_{5}\right) & =e_{1}+2 e_{3}-e_{6}, \\
u_{2}=\frac{1}{2}(g-\mathbf{1})\left(e_{3}+2 e_{4}+2 e_{5}\right) & =e_{2}-e_{3}+e_{6}, \\
u_{3}=1 & =e_{4}+e_{6}, \\
u_{4}=\frac{1}{2}(g-\mathbf{1})\left(e_{5}\right) & =e_{5}-2 e_{6} .
\end{array} \quad \Phi=\left(\begin{array}{cccc}
4 & 0 & 2 & -4 \\
-2 & 1 & -2 & 2 \\
0 & 0 & 0 & 1 \\
0 & 2 & 3 & -5
\end{array}\right) .\right.
$$

Let us first experiment with the left side algorithm. We begin with $\dot{v}_{1}=u_{1}$, and the basis $\left(\dot{v}_{1}, u_{2}, u_{3}, u_{4}\right)$ of $S$. We calculate $y_{1}=\xi_{1} \dot{v}_{1}+u_{2}$ with the condition $\phi\left(\dot{v}_{1}, y_{1}\right)=0$; immediately, we obtain $y_{1}=u_{2}$. We begin the second step with $v_{1}=u_{1}$, $\dot{v}_{2}=u_{2}$, and the basis $\left(v_{1}, \dot{v}_{2}, u_{3}, u_{4}\right)$. We calculate $y_{1}=\xi_{1} v_{1}+\xi_{2} \dot{v}_{2}+u_{3}$ with the conditions $\phi\left(v_{1}, y_{1}\right)=\phi\left(\dot{v}_{2}, y_{1}\right)=0$, which give the equations $4 \xi_{1}+2=-2 \xi_{1}+\xi_{2}-2=0$, whence $\xi_{1}=-1 / 2, \xi_{2}=1$, and $y_{1}=-\frac{1}{2} u_{1}+u_{2}+u_{3}$. Unfortunately, $\phi\left(y_{1}, y_{1}\right)=0$ and we must calculate also $y_{2}=\xi_{1} v_{1}+\xi_{2} \dot{v}_{2}+u_{4}$; the equations $4 \xi_{1}-4=-2 \xi_{1}+\xi_{2}+2=0$ give $\xi_{1}=1$, $\xi_{2}=0$ and $y_{2}=u_{1}+u_{4}$. Since $\phi\left(y_{2}, y_{2}\right)=-5$, we begin the third step with $v_{1}=u_{1}, v_{2}=u_{2}$ and $\dot{v}_{3}=u_{1}+u_{4}$. In this final step, we calculate $y_{1}=\xi_{1} v_{1}+\xi_{2} v_{2}+\xi_{3} \dot{v}_{3}+u_{3}$; the wanted conditions give the equations

$$
4 \xi_{1}+2=-2 \xi_{1}+\xi_{2}-2=4 \xi_{1}+2 \xi_{2}-5 \xi_{3}+5=0 ;
$$

consequently, $\xi_{1}=-1 / 2$ and $\xi_{2}=\xi_{3}=1$. Here is the resulting basis $\left(v_{1}, \ldots, v_{4}\right)$ and the matrix $\Phi^{\prime}$ of $\phi$ in this basis:

$$
\left\{\begin{array}{l}
v_{1}=u_{1}, \\
v_{2}=u_{2}, \\
v_{3}=u_{1}+u_{4}, \\
v_{4}=\frac{1}{2} u_{1}+u_{2}+u_{3}+u_{4},
\end{array} \Phi^{\prime}=\left(\begin{array}{cccc}
4 & 0 & 0 & 0 \\
-2 & 1 & 0 & 0 \\
4 & 2 & -5 & 0 \\
0 & 3 & 2 & 1
\end{array}\right)\right.
$$

We have $g=\mathrm{R}\left(v_{1}\right) \mathrm{R}\left(v_{2}\right) \mathrm{R}\left(v_{3}\right) \mathrm{R}\left(v_{4}\right)$ with $v_{1}=e_{1}+2 e_{3}-e_{6}, \quad v_{2}=e_{2}-e_{3}+e_{6}, \quad v_{3}=e_{1}+2 e_{3}+e_{5}-3 e_{6}, \quad v_{4}=$ $\frac{1}{2} e_{1}+e_{2}+e_{4}+e_{5}-\frac{1}{2} e_{6}$.

Now let us experiment with the disordered algorithm that gives the vectors of a triangularizing basis in the disorder $\left(v_{1}, v_{4}, v_{2}, v_{3}\right)$. To take advantage of the vanishing of $\phi\left(u_{4}, u_{1}\right)$, we begin with $\dot{v}_{1}=u_{4}$, the basis $\left(\dot{v}_{1}, u_{1}, u_{2}, u_{3}\right)$ and $y_{1}=\xi_{1} \dot{v}_{1}+u_{1}$; the condition $\phi\left(\dot{v}_{1}, y_{1}\right)=0$ gives immediately $y_{1}=u_{1}$. Therefore, we start the second step with $v_{1}=u_{4}$, 
$\dot{v}_{4}=u_{1}$ and with the basis $\left(v_{1}, u_{2}, u_{3}, \dot{v}_{4}\right)$; we calculate $y_{1}=\xi_{1} v_{1}+u_{3}+\xi_{2} \dot{v}_{4}$ with the conditions $\phi\left(v_{1}, y_{1}\right)=\phi\left(y_{1}, \dot{v}_{4}\right)=0$. The resulting equations $-5 \xi_{1}+3=4 \xi_{2}=0$ give $\xi_{1}=3 / 5, \xi_{2}=0$ and $y_{1}=u_{3}+\frac{3}{5} u_{4}$, whence $\phi\left(y_{1}, y_{1}\right)=3 / 5$. Therefore, we start the third (and last) step with $v_{1}=u_{4}, \dot{v}_{2}=u_{3}+\frac{3}{5} u_{4}, v_{4}=u_{1}$, and with the basis $\left(v_{1}, \dot{v}_{2}, u_{2}, v_{4}\right)$. We calculate $y_{1}=\xi_{1} v_{1}+\xi_{2} \dot{v}_{2}+u_{2}+\xi_{3} v_{4}$ with the conditions $\phi\left(v_{1}, y_{1}\right)=\phi\left(\dot{v}_{2}, y_{1}\right)=\phi\left(y_{1}, v_{4}\right)=0$, which give the equations

$$
-5 \xi_{1}+2=-2 \xi_{1}+\frac{3}{5} \xi_{2}+\frac{6}{5}=-2+4 \xi_{3}=0 ;
$$

consequently, $\xi_{1}=2 / 5, \xi_{2}=-2 / 3, \xi_{3}=1 / 2$. Here is the resulting basis $\left(v_{1}, v_{2}, v_{3}, v_{4}\right)$, and the matrix of $\phi$ in this basis:

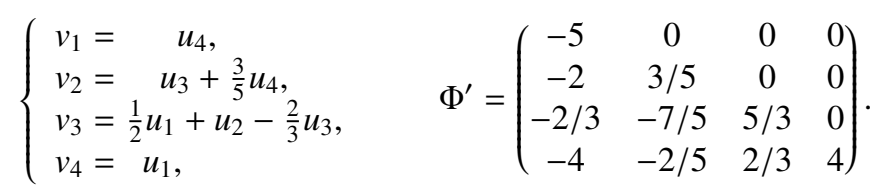

Thus $g=\mathrm{R}\left(v_{1}\right) \mathrm{R}\left(v_{2}\right) \mathrm{R}\left(v_{3}\right) \mathrm{R}\left(v_{4}\right)$ with $v_{1}=e_{5}-2 e_{6}, v_{2}=e_{4}+\frac{3}{5} e_{5}-\frac{1}{5} e_{6}, v_{3}=\frac{1}{2} e_{1}+e_{2}-\frac{2}{3} e_{4}-\frac{1}{6} e_{6}, v_{4}=e_{1}+2 e_{3}-e_{6}$. To compare these two versions, we compare the square matrices associated with the systems of equations (7.1) and (7.2):

$$
\left(\begin{array}{ccc}
4 & 0 & 0 \\
-2 & 1 & 0 \\
4 & 2 & -5
\end{array}\right) \quad \text { and } \quad\left(\begin{array}{ccc}
-5 & 0 & 0 \\
-2 & 3 / 5 & 0 \\
0 & 0 & 4
\end{array}\right)
$$

The first matrix is just a lower triangular matrix, with 6 meaningful entries. Along the diagonal of the second matrix, there is a lower triangular submatrix of order 2 , and a submatrix of order 1 which would appear to be upper triangular if it were larger; the main fact is that the second matrix contains only 4 meaningful entries. For a space $S$ of arbitrary dimension $s$, the calculation is shorter if we calculate the vectors of a triangularizing basis $\left(v_{1}, \ldots, v_{s}\right)$ in this disorder: firstly $v_{1}$ and $v_{s}$ (either $\left(v_{1}, v_{s}\right)$ or $\left.\left(v_{s}, v_{1}\right)\right)$, secondly $v_{2}$ and $v_{s-1}$ (either $\left(v_{2}, v_{s-1}\right)$ or $\left.\left(v_{s-1}, v_{2}\right)\right)$, thirdly $v_{3}$ and $v_{s-2}$, and so forth...

\section{References}

Chevalley, C. (1954). The algebraic theory of spinors, New York, Columbia Univ. Press. Reprinted in Chevalley C. (1997), Collected works, 2, Springer.

Dieudonné J. (1958). Sur les groupes classiques, Paris, Hermann.

Helmstetter J. (2017?). Bilinear forms derived from lipschitzian elements in Clifford algebras. In preparation.

Helmstetter J., \& Micali A. (2008). Quadratic mappings and Clifford algebras, Basel, Birkhäuser.

\section{Copyrights}

Copyright for this article is retained by the author(s), with first publication rights granted to the journal.

This is an open-access article distributed under the terms and conditions of the Creative Commons Attribution license (http://creativecommons.org/licenses/by/4.0/). 